TITLE:

\title{
Spontaneous Emission of Plasma Waves in the Presence of a Finite Amplitude Wave( Dissertation_全文 )
}

AUTHOR(S):

Katayama, Yoshishige

\section{CITATION:}

Katayama, Yoshishige. Spontaneous Emission of Plasma Waves in the Presence of a Finite Amplitude Wave. 京都大学, 1973, 理学博士

ISSUE DATE:

1973-03-23

URL:

https://doi.org/10.14989/doctor.k1304

RIGHT: 


$$
\text { 学位申請椧文 }
$$


Spontaneous Emission of Plasma Waves

In the Presence of a Finite Amplitude Wave

Yoshishige Katayama

Department of Physics, Faculty of Science

Kyoto University, Kyoto 


\section{1}

Introduction and summary

In 1929, Iangmuir and Tonks found a systematic oscillation in a plasma and showed that lis angular frequency is given by $\omega_{p}=\left(\left(4 \pi n_{0} e^{2}\right) / m\right)^{1 / 2}$, where $m$, e and $n_{0}$ are the mass, charge and average density of the particle. Since then, this oscillation has been studied in many aspects both experimentally and theorectically. The first theoretical work is the one by Vlasov ${ }^{1}$, who proposed to describe wave processes in a rarefied plasma by means of a kinetic equation with a self-consistent field, starting from the collisionless Boltzmann equation and the Poisson equation. After his theory, Landau pointed out the mathematical error of his treatment and then theoretically predicted the phenomenon of Landau damping 2$)$. Both treatments are based on the theory linear with respect to the fluctuation field. If we go beyond the linear theory, however, those particles whose velocities are close to the phase velocity of the wave continue to be accelerated (or decelerated) by the wave field and show a secular behavior in time. As a result, there is a time above which the ilnear theory becomes invalid. Let us consider two time scales. One is the Landau damping time of the wave, (to be referred to as $T_{D}$ ) and the other 1s the period of bouncing motion of a particle in the bottom of potential trough of the wave (referred as $\tau_{\beta}$ ). 
The latter time scale is inversely proportional to the square root of the field strength of the wave. When the amplitude of the wave field is suffictently small, so that $\tau_{D} \ll \tau_{B}$, we can use the 11near theory in respect of the interaction between the wave and particles. As the amplitude of the wave becomes larger and $\tau_{\beta}$ gets nearly equal or larger than $\tau_{D}$, the nonlinear coupling of the wave and particles begins to play lmportant roles. Such a situation was first discussed by Bohm and Gross ${ }^{3}$. They divided the partioles into two groups: one is composed of the particles which are trapped in the potentialenergy troughs of the wave and which are necessary to be treated nonlineariy, and the other is composed of those which are untrapped. These authors have studied the relation between the distribution of the two groups of particles and the stationary character of the wave. As an extension of their work, a theory is developed by Bernstein, Greene and Kruska ${ }^{4)}$ who discussed a stationary exact solution of the nonlinear Vlasov equation. The stability of the solution was not discussed, however. There are other nonlinear theories of plasmas, for instance; the weak turbulence theory, the orbit-modification theory, and sơn. We shall not refer to these theorles here.

Experimentally, a considerable progress has been made In plasma physics since 1955 and we are now able to 
produce very quiescent plasmas in laboratorles and also to exclte waves of arbitrary frequencies by applying high frequency oscillations to a Langmuir prabe. As a result the dispersion relation for plasma waves have been studied in detail and many of the results of the inear theory have been experimentally checked. In particular, the phenomenon of Landau damping has been observed by Malmberg, Wharton and Drumond ${ }^{5)}$. Further progress in experimental techniques has made it possible to excite a large-amplitude coherent wave for which the relation $\tau_{\beta}>\tau_{D}$ holds. The phenomena due to the presence of trapped particles have been w1dely studied both experimentally and theoretically. Among them, the phenomena of the amplitude oscillation and the trapped-particle instability are noticeable. The former is a phenomenon that the amplitude of a large-amplitude wave (hereafter referred to as the carrier wave) excited In a plasma oscillates in space with a period approximately equal to $v_{p} \tau_{B}$ ( $v_{p}$ is the carrier phase velocity) and was observed by Malmberg and Wharton ${ }^{6)}$ for the first time. The particles trapped in the potential troughs of the wave make the bouncing motion and, therefore, they periodically exchange their energies with the carrier wave. It is the physical reason for this phenomenon. Theoretically, it has been investigated by $\mathrm{O}^{\prime} \mathrm{Ne} 11^{8}$ ) and by $\mathrm{Al}$ 'thul and Karpman" using different methods. The trapped particle 
instability is a phenomenon in whlch waves of frequencles different from the carrier-wave frequency by approximately Integral multiples of the bounce frequency $2 \pi / \tau_{B}$ (1.e. sideband waves to the carrier) become unstables, and has been observed by Wharton ${ }^{7)}$ et al. This phenomenon is due to the energy transfer from the trapped particles bouncing In the periodic potential troughs of the carrier wave to sideband waves via resonance coupling and it hes been studied theoretically by Kröer-Dawson-Sudan ${ }^{18}$ ) and Mima-Nishikawl(p). This problem has raised a question regarding the stability of the B-G-K solution mentioned above.

The above problems are all concerned with the phase coherent response of a plasma to the electric field. In plasmas, however, there also exists spontaneous emission of plasma waves. It comes from the discreteness of the plasma, namely, charged particles moving in the dielectric medium emit collective oscillations by Cerenkov emission. The fluctuation level in a thermalequilibuium plasma is derivable from a balance between the rate of spontaneous emission and that of Landau damping. This corresponds to the fluctuation dissipation theorem. In a homogeneous plasma, the emission rate can be calculated by making use of a particle orbit of free motion and a dlelectric function derived by the linear theory. In an inhomogeneous system where a large-amp11tude 
coherent wave is propagating, however, both of the particle orbit and the dielectric character of the plasma are modified by the existence of the carrier wave. The principal subject of this work is to develop a theory of the spontaneous Cerenkov emission in which the effects of the carrier wave mentioned above are taken into account.

The main motivations of this work are the following. First, the theory of a moderately strong lon-wave turbulence given by N1shikawa and $\mathrm{wu}^{12)}$ indicates that in collisionless plasmas, particle-trapping (discussed in a statistical sense) due to low-level ion-wave fluctuations could be signiflcant even for stable plasmas. Accoralng to their considerations, it may be necessary to take into account the effect of electron-trapping for the spontaneous emission even in the discussion of the thermal level of ion-wave fluctuations. The second motivation is connected with the work on the anomalous resistivity by Dupree ${ }^{13)}$, Kadomtsev and Pogutse $\mathrm{e}^{14)}$ and others. 15) According to the theory of Balescu ${ }^{16)}$, Lenard ${ }^{17)}$, and Guernsey ${ }^{18}$ ) on the collisions between the particles and waves in a plasma, there exists an effect of the slowing down of the particles aue to the reaction of the medium against the spontaneous Cerenkov emission by the particles. Dupree and others proposed that this slowing-down effect would become much larger if we take into account the effect of coherent emission due to the localization of the 
particles in phase-space (Dupree called it "macroparticle"), and consequentiy, 1ts effect would give rise to an anomalous effective collision frequency on the particles. Following their proposal, we want to consider the influence of the carrier wave on the spontaneous Cerenkov emission and the coherence effect of this emission due to the existence of the carrier wave. Finally, although the experimentaily observed sideband excitation in the presence of a large-amplitude wave $1 \mathrm{~s}$ considered to be explained by the theory of trapped particle instabilities, there also exists a possibility of spontaneous emission of sideband waves as a result of a periodic modulation of the particle orbits. For a complete comparison of the theory with experiment, it is necessary to clarify a qualitative difference between the sideband excltation via trapped particle instability and that via spontaneous emission.

Let us present a brief summary of the results obtained In this work. In the case of the emission of an untrapped particle, waves of many different frequencies are emitted. They include the one which satisfles the ordinary Cerenkov condition, but its emission rate 1s weakened as compared with the emission by a freely-moving particle. Also included 1s an emission corresponding to the scattering of the carrier wave by the particle (for example, the Thomson scattering). A trapped particle emits sideband waves in addition to the carrier wave itself. The frequencies of 
the sideband waves are different from the carrier-wave frequency by approximately integral multiples of the bounce frequency and the spectrum of these emissions in the wavenumber space becomes discrete and symmetric around the wave-number of the carrier wave. The effect of the presence of the carrier wave on the dielectric proparty of the system appears mainly in the emission by the trapped particle. Namely, the dispersion relation and emission rate of the sideband waves are modified in comparison with the result of the primary treatment where this effect is neglected. For the emission by an aggregate of trapped particles, there appears a coherence effect because of the localization of particles in the potential trough and also because of a periodic array of groups of localized particles which reflects the periodicity of the carrier-wave potential. These effects occur only for the emission of the carrier wave itself.

We discuss the problem of spontaneous emission of plasma waves in the presence of a finite-amplitude wave, dividing the consideration into two parts. In the first, the theory of the spontaneous emission in a homogeneous plasma is briefly reviewed. We also refer to a theory ${ }^{19}$ ) which takes into account only the effect of the orbit modification, but neglects the effect of the carrier wave on the dielectric character of the plasma. Such a theory may be used in the case when the amplitude of the carrier 
wave is sufficlently small. In the second part, we develop a theory which takes into account the effect of the carrier wave on the dielectric character of the plasma as well as on the particle orbit. The theory is based on the Klimontovich formallsm 20 ) and is an extension of the theory of spontaneous emission in a uniform plasma to an inhomogeneous plasma. 
References:

1) A. A. Vlasov: Zh. Eksp. Teor. Fiz. $\underline{8}$ (1938) 291 or Usp. F1z. Nauk 93 (1967) 444 [Sov. Phys. Usp. 10 (1968) $721]$.

2) I. D. Landau: 2h. Eksp. Teor. F1z. 16 (1946) or Usp. F1z. Nauk 93 (1967) 527.

3) D. Bohm and E. P. Gross: Phys. Rev. 75 (1949) 1851.

4) I. B. Bernstein, J. M. Greene and M. D. Kruskal: Phys. Rev. 108 (1957) 546.

5) J. H. Malmberg, C. B. Wharton and W. E. Drummond: Plasma Physics and Controlled Nuclear Fusion Research, Vol. 1, Vienna, 1966, p485.

6) J. H. Malmberg and C. B. Wharton: Phys. Rev. Letters 19 (1967) 775 .

7) C. B. Wharton, J. H. Malmberg and T. M. O'Ne1l: Phys. Fluids I1 (1968) 1761.

8) T. M. O'Ne1l: Phys. Fluids $\underline{8}$ (1965) 2255.

9) I. M. Al'thul and N. I. Karpman: Soviet Physics-JETP 22 (1966) 361 .

10) W. L. Kruer, J. M. Dawson and R, N. Sudan: Phys, Rev. Letters 23 (1969) 838.

11) K. Mima and K. Nishikawa: J, Phys. Soc. Japan 30 (1971) 1722 .

12) K. N1sh1kewa and C. S. Wu: Phys. Rev, Letters $\underline{23}$ (1969) 1020 . 
13) T. H. Dupree: Phys. Rev. Letters 25 (1970) 789.

14) B. B. Kadomtser and O. Pogutse: Phys. Rev. Letters 25 (1970) 1155.

15) B. Coppi and F. Santin1: Phys. Rev. Letters 28 (1972) 19.

16) R, Balescu: Phys. Fluids $\underline{3}$ (1960) 52 .

17) A. Lenard: Ann. Phys. (N.Y.) 10 (1960) 390.

18) R. L. Guernsey: dissertation, University of Michigan, 1960 (umpublished).

19) Y. Katayama: J. Phys. Soc. Japan 31 (1971) 959.

Kakuyugo-Kenkyu, C1rcular in Japan 26 (Aug. 1971) 320.

20) This part of the work is appeared in

J. Phys. Soc. Japan 33 (1972) 483. 
Part I

81. Spontaneous Emissions in Plasmas

First of all, let us derive a general expression for the rate of spontaneous Cerenkov emission of longitudinal waves per unit time by a single particle. This rate is equal to the work done by the medium as a reaction to the wave emission by the particle because of the energy conservation in the process of the emtssion. Therefore, the rate of wave emission, denoted by $I_{\sigma}$, is given by

$$
I_{\sigma}=-\int d \vec{r} \vec{j}_{\sigma}(r, t) \cdot \vec{E}_{\sigma}(r, t),
$$

where $\sigma$ represents the species of the particle, and $\vec{f}_{\sigma}$, $\overrightarrow{\mathrm{E}}_{\sigma}$ are the current dens1ty corresponding to the particle motion and the electric fleld due to the particle, respectively. The integration is carried out over the whole plasma. Let us Fourier-analyse in space and time as

$$
\vec{\jmath}_{\sigma}(\vec{r}, t)=\int d \vec{k} d \omega \overrightarrow{J_{\sigma}}(\vec{k}, \omega) e^{i \vec{k} \cdot \vec{r}-1 \omega t}
$$

and

$$
\vec{E}_{\sigma}(\vec{k}, t)=\int d \vec{k} d \omega \vec{E}_{\sigma}(\vec{k}, \omega) e^{i \vec{k} \cdot \vec{k}-1 \omega t}
$$

Then, $I_{\sigma}$ is written in the Fourier representation as 


$$
I_{\sigma}=-(2 \pi)^{3} \int d \vec{k} d \omega d \omega \cdot \vec{E}_{\sigma}(\vec{k}, \omega) \cdot \vec{j}_{\sigma}\left(-\vec{k}, \omega^{+}\right) e^{-1\left(\omega+\omega^{\prime}\right) t} .
$$

By means of the test-particle method, we can relate $\vec{\jmath}_{\sigma}$ and $\vec{E}_{\sigma}$ with the charge density $p_{\sigma}$ corresponding to the moving particle. The Polsson equation in the Fourier representation is given by

$$
\overrightarrow{1 k} \cdot \vec{D}_{\sigma}(\vec{k}, \omega)-4 \pi \rho_{\sigma}(\vec{k}, \omega)
$$

where $\vec{D}_{\sigma}$ is the corresponding electric displacement. The electric displacement is related to the electric fleld as

$$
\vec{D}_{\sigma}(\vec{k}, \omega)=\stackrel{\Leftrightarrow}{e}(\overrightarrow{\vec{k}}, \omega) \overrightarrow{E_{\sigma}}(\vec{k}, \omega)
$$

where $(\vec{k}, w)$ is the dielectric tenser. In this treatment, however, we use the dielectric function for a homogeneous and isotropic plasma since we suppose that the amplitude of the carrier wave is smail enough for its effect on the dielectric property of the system to be negligible. From the equation of continulty, $\vec{j}_{\sigma}(\vec{k}, w)$ is given by

$$
\vec{k} \cdot \vec{j}_{\sigma}(\vec{k}, \omega)=\omega \rho_{\sigma}(\vec{k}, \omega)
$$


By making use of eqs.(1.3) (1.6), the expression for $I_{\sigma}$ is rewritten as

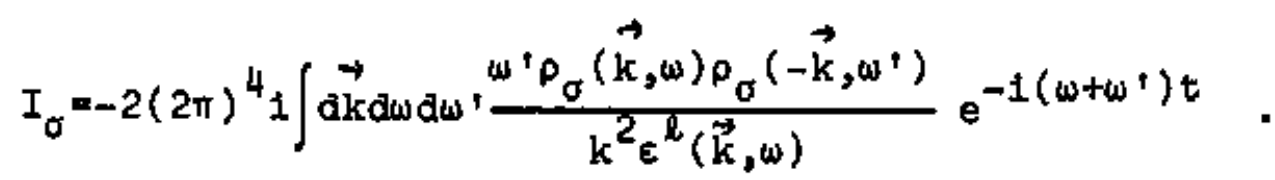

We appiy eq.(1.7) to the case of a homogeneous unmagnetized plasma. In this case, we may adopt the orbit of free motion for the test particle. The charge density corresponding to this motion $1 \mathrm{~s}$

$$
\rho(r, t)=e \delta\left(\vec{r}-\vec{v}_{0} t\right)
$$

$\overrightarrow{\mathrm{v}}_{0}$ belng the unperturbed velocity of the particle. Substituting the Fourler component of this quantity into eq.(1.7), we obtain the emission rate of the particle with the velocity $\vec{v}_{0}$ as

$$
I\left(\vec{v}_{0}\right)=-\frac{4 \pi e^{2}}{(2 \pi)^{3}} \int \overrightarrow{d \vec{k}} \frac{\vec{k} \cdot \vec{v}_{0}}{k^{2}} \operatorname{Im} \frac{j}{\varepsilon^{l}(\vec{k}, \omega)} .
$$

This expression can approximateiy be written as

$$
I\left(\vec{v}_{0}\right)=\frac{e^{2}}{(2 \pi)} \int d \vec{k} \frac{\omega_{k}}{k^{2}\left(\left.\frac{\partial E^{e}}{\partial \omega}\right|_{\omega=\omega_{k}}\right)} \delta\left(\vec{k} \cdot \vec{v}_{0}-\omega_{k}\right),
$$

where $\omega_{k}$ satisfies the equation $\operatorname{Re} e^{e}\left(\vec{k}, \omega_{k}\right)=0$. 
32. Effects of a Finlte Amplitude Wave

We consider the spontaneous Cerenkov emission in an unmagnetized plasma in the presence of a finite-amplitude wave. For simplicity, we confine ourselves to a onedimensional treatment. The equation of motion for the test-particle is

$$
m \frac{d^{2} x}{d t^{2}}--e E_{0} \sin \left(k_{0} x-\Omega_{0} t\right)
$$

where $E_{0} \sin \left(k_{0} x-\Omega_{0} t\right)$ represents the carrier wave with the amplitude $E_{0}$, the frequency $\Omega_{0}$ and the wave number $k_{0}$. Considering this equation in the wave frame, we find that the total energy of the particle $W$ is conserved in this frame and the whole particles can be d1vided into two groups according to their total energles; one is a group of untrapped particles whose total energies are larger than $\mathrm{eE}_{0} / \mathrm{k}_{0}$ and the other is a group of trapped ones whose total energies are smaller than $e \mathrm{E}_{0} / \mathrm{k}_{0}$. Therefore, we separate that problem Into two cases as follows

(1) Untrapped-particle case:

For simplicity, we consider only those particles whose kinetic energies are much larger than their potential energies. We then adopt the following orbit for the testparticle in the laboratory frame:

$$
x(t)=\alpha \sin \left(k_{0} x-\Omega_{0} t\right)+v_{0} t,
$$


where $x_{0}$ and $v_{0}$ are the initial position and velocity of the test particle and

$$
\begin{aligned}
& \alpha \equiv \frac{\frac{e}{m} E_{0}}{\left(k_{0} v_{0}-\Omega_{0}\right)^{2}} \\
& \Omega_{D} \equiv \Omega_{0}-k_{0} v_{0}
\end{aligned}
$$

This orbit is correct to the first order with respect to the expansion parameter $\mathrm{eE}_{0} / \mathrm{k}_{0} \mathrm{~W}$. The charge density corresponding to this motion is given by

$$
\rho(x, t)=\operatorname{eo}\left(x-v_{0} t-a \sin \left(k_{0} x_{0}-\Omega_{D} t\right)\right)
$$

Substituting the Fourier component of this quentity into eq.(1.7) and averaging over the intial position, we obtain the emission rate by the untrapped particle after some calculations as

$$
I^{W T}\left(v_{0}\right)=2 \pi e^{2} \sum_{n} \int \frac{\omega_{k}}{k^{2}\left(\left.\frac{\partial \varepsilon}{\partial \omega}\right|_{\omega=\omega_{k}}\right)} J_{n}^{2}(k \alpha) \delta\left(\omega_{k}-k v_{0}-n \Omega_{D}\right),
$$

where $I_{n}$ is the Bessel function. In the above expression, the term $n=0$ shows that the wave which satisfies the ordinary cerenkov condition is emitted but its rate is reduced by a factor $J_{0}^{2}(k+x)$. This reduction is aue to the fact that the particle velocity can be equal to the 
phase velocity of the modulation of the particle orbit by the wave fleld. The term $n=1$ corresponds to the Thomson scattering of the wave $\left(k_{0}, n_{0}\right)$ by the particle of velocity $\boldsymbol{V}_{0}$ as seen by the resonance condition $\omega_{k}-\Omega_{0}=\left(k-k_{0}\right) v_{0}$.

\section{(i1) Trapped-particle case:}

For simpliclty we confine our treatment only to those particles which are trapped in the bottom of the potential trough. We obtain the orblt for such a particle perturbationally as

$$
x(t)=V_{0}(t)+\frac{1}{\omega_{B}} \sqrt{\frac{2 W}{m}} \sin \left(\omega_{0} t+\beta\right),
$$

where $v_{0}$ is the phase velocity of the carrier wave, $\omega_{0}$ is the bounce frequency in the bottom of the potent1al trough and $B$ is the initial phase of the particle. The above expression is correct to the first order with respect to the expansion parameter $\mathrm{k}_{0} \mathrm{~W} / \mathrm{eE_{0 }}$. The corresponding charge density is given by

$$
\rho(x, t)=e \delta\left(x-V_{0} t-\frac{1}{\omega_{B}} \sqrt{\frac{2 W}{m}} \sin \left(\omega_{B} t+B\right)\right) .
$$

The same procedure as done for the case of the untrapped particle brings the final expression for the emission rate of the trapped particle with the total energy $W$ to the form: 


$$
I^{T}(W)=2 \pi e^{2} \sum \int d k \frac{\omega_{k}}{k^{2}\left(\left.\frac{\partial \varepsilon}{\partial \omega}\right|_{\omega=\omega_{k}}\right)} J_{n}^{2}\left(\frac{k}{\omega_{B}} \sqrt{\left.\frac{2 W}{m}\right) \delta\left(\omega_{k}-k V_{0}-n \omega_{B}\right)} .\right.
$$

In this case, the term $n=0$ corresponds to the emission under the ordinary Cerenkov condition. The emission rate 1s, however, reduced by a factor $\mathrm{J}_{0}^{2}\left(\frac{\mathrm{k}}{\mathrm{w}_{\mathrm{B}}} \sqrt{\frac{\sqrt{\mathrm{W}}}{\mathrm{m}}}\right)$ compared with that of a freely moving particle. The resonance condition for the term nło shows that the trapped particles also emit side-band waves whose frequencies are different from the carrier-wave frequency by approximately integral multiples of $\omega_{B}$ and consequently, the spectrum of emission becomes discrete in $k$ space. These situations reflect the bouncing motion of the particle in the potential well. If we treat the motion of trapped particles more exactly, however, the bounce frequency Is no longer independent of the particle energy and the result mentioned above would be modified correspondingly. We neglect the particles which exist near the boundary between the trapped and untrapped reglons. We find, however, from the equation of motion that these particles spend almost all of their time near the top of the potential with the velocity nearly equal to the phase velocity of the carrier wave. Therefore, they will emit the carrier wave on the average as a freely moving particle with the velocity $v_{0}$ does. 


\section{$\$ 3$ Coherence effect}

In the present system the trapped particles are localized to each potential trough because of the existence of the carrter wave. Consequently, the coherence effect can occur for the case of the emission by an aggregate of trapped particles. There exists another coherent effect due to a perlodic array of groups of localized particles which reflects the periodiclty of the carrier-wave potential. Let us consider these coherence effects in the following. We specify the trapped particie with a pair of suffixes $(J, p)$, which denotes the p-th particle trapped in the J-th potential trough. The Fourler component of the charge density corresponding to the particle $(J, p)$ is

$$
\rho^{(J, p)}(k, \omega)=\frac{e}{2 \pi} \sum_{n} J_{n}\left(\frac{k}{\omega_{B}} \sqrt{\frac{2 W(J, p)}{m}}\right) e^{-i n B(J, p)} \delta\left(\omega-k V_{0}-n \omega_{B}\right) e^{-1 k x_{J}}
$$

where $W(J, p)$ and $B(J, p)$ are the total energy and Intial phase of the particle $(J, p)$ in the wave frame respectively and $x_{J}$ is the initial position of the bottom of the J-th potential trough. The charge density corresponding to an aggregate of trapped particles is obtained by summing eq.(3.1) over $(\mathrm{J}, \mathrm{p})$. Making use of eq. (1.7), we get the expression for the emission rate by alI of the trapped particles as 


$$
\begin{aligned}
& I^{T}=2 \pi e^{2}\left(\sum_{(J, p)} \sum_{n} \int d k \frac{\omega_{k}}{k^{2}\left(\left.\frac{\partial \varepsilon}{\partial \omega}\right|_{\omega=\omega_{k}}\right)} J_{n}^{2}\left(\frac{k}{w_{B}} \sqrt{\frac{(2 W(J, p)}{m}}\right) \delta\left(\omega_{k}-k V_{0}-n \omega_{B}\right)\right.
\end{aligned}
$$

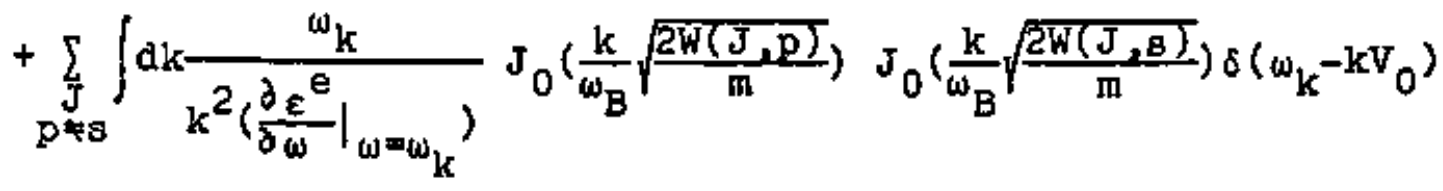

$$
\begin{aligned}
& +\sum_{\substack{j \neq L \\
p, s}} \int \frac{\omega_{k}}{k^{2}\left(\left.\frac{\partial \varepsilon}{\partial \omega}\right|_{w \omega_{k}} ^{e}\right)} J_{0}\left(\frac{k}{\omega_{B}} \sqrt{\left.\frac{2 W\left(J_{2} D\right)}{m}\right)} J_{0}\left(\frac{k}{\omega_{B}} \sqrt{\frac{2 W\left(L_{2} s\right)}{m}}\right)\right. \\
& \delta\left(\omega_{k}-k v_{0}\right) e^{-1 k\left(x_{J}-x_{L}\right)}
\end{aligned}
$$

Let us discuss each term of the right hand side of the above expression separately.

\section{(1) First term:}

This term is the simple sum of the result for a single particle obtained in $\$ 2$, and represents an ordinary incoherent contribution. Let us consider the emission by the aggregate of particles trapped in the same potential and denote the number of them as $\mathrm{N}_{\mathrm{J}}$. Then, the emission rate corresponding to this term (denoted by $I_{1}^{T}$ ) is $N_{J}$ times as large as that of a single particle if we neglect the difference of the energy between different particles:

$$
I_{I}^{T}-N_{J} I_{\mu}^{T}
$$


where $I_{j}^{T}$ is the emission rate by a single particle.

(1i) second term:

This term represents the coherent contribution of the group of particles in the same potential trough, and arises only for the emission of the carrier wave 1tself. The order of magnitude of this contribution (denoted by $I_{2}^{T}$ ) is $N_{J}^{2}$ times as large as $I_{\mu}^{T}$ :

$$
I_{2}^{T}-N_{J}^{2} I_{\mu}^{T}
$$

\section{(111) third term:}

Whis term corresponds to the coherence effect between the emissions by the particles trapped in different potential troughs. As seen from the factor $\exp \left[-1 k\left(x_{J}-x_{L}\right)\right]$, this effect appears only for the emission of the carrier wave 1tself.

If we treat the problem three-dimensionally, these coherence effects will considerably be reduced except for the emission of the wave whose direction of propagation is equal to that of the carrier wave, since the localization of trapped particles mentioned above is essentially one-demensional and so there is no locajization over the directions perpendicular to the wave vector of the carrier wave. 
Part II

Synopsis

The spontaneous Cerenkov emission of longltudinal waves In a plasma with small but finite amplitude wave (hereafter referred as the carrier wave) 1s investigated. Two effects of the carrier wave are taken into account; one is the modification of the test-particle orbit and the other that of the dielectric character of the plasma. It is shown that particles trapped in the potential-energy troughs of the carrier wave emit a discrete spectrum of sldeband waves (In addition to the carrier wave 1tself) whose frequencles are different from the carrier-wave frequency by approximately integral multiples of the bounce frequency. An untrapped particle also emtts waves at several different frequencles, including the one which satisfies the ordinary Cerenkov condition, but these frequencies depend on the particle velocity. The results are compared with those reported in our previous paper where only the effect of the orbit modification is taken into consideration. 


\section{\$1. Introduction}

In the usual treatment ${ }^{1)}$ of the spontaneous Cerenkov emission of longltudinal waves, the plasma is assumed to be spatially homogeneous and a particle orbit of free motion is used for the test particle. In this paper, we consider the situation in whlch a finite-amplitude wave (hereafter to be referred to as the carrier wave) is stationarily traveling in the plasma. Two effects of the carrier wave are to be taken into consideration; one is the modification of the test-particle orbit and the other is that of the dielectric character of the plasma.

We have recently presented a theory 2 ) in which only the modification of the test-particle orbit is taken into account, the dielectric character being assumed to be the same as that for a homogeneous system. We found that 1) the particles trapped at the bottoms of the potentlal-energy troughs of the carrier wave emit the carrier wave itself, but at the same time its sideband waves whose frequencies are different from the carrier frequency by the 1ntegral aultiples of the bounce frequency of the trapped particles, and 11) an untrapped particle also emits waves at several different frequencles, inclualng the one which satisfies the ordinary Cerenkov condition, but these frequencles depend on the velocity of the test part1cle. 
In the present paper, we take into account the effect on the dielectric character of the plasma as well as that on the test-particle orbit. Two important modifications to our previous result ${ }^{2}$ ) are obtained regarding the emission of sideband waves by the trapped particles. First, the dispersion relation (the frequency versus navenumber relation) of sideband waves is modified from the one in a homogeneous system. Secondly the emission rates of the sldeband waves are modified. In addition to the sidebands the trapped particles also emit the carrier wave itseif. For the emission by the untrapped particles, there is no substantial modification by the change in the dielectric character of the system.

We start from the assumption that there exists a so-called B.G.K. state 3) 4). Although the stability of a B.G.K. state is open to question ${ }^{5)}$, we do. not concern this problem. In ract, our results can equadly be used for both stable and weakly unstable systems, as in the case of a homogeneous system ${ }^{6)}$. We then consider a microscopic charge-density fluctuation to this B.G.K. state and calculate the electric field induced by this fluctuation. This is done in $\$ 2$ and 83. The relation between the electric field and the charge-density fluctuation is expressed in terms of the generalized susceptibilities of the plasma which are calculated in 54 . The spontaneous Cerenkov emission rate is then calculated in 55 with the use of a kinetic wave equation for the spectral density of the electric fleld. The results are discussed in the last section. 


\section{Basic Equations}

For simplicity, we consider a one-dimensional and non-magnetized plasma. We confine our attention to high-frequency electrostatic waves and regard the ions as a uniform background of positive charge.

In order to discuss spontaneous cerenkov emission, the contribution from microscopic fluctuations must be taken into account in the kinetic wave aquation. Therefore, we start from the klimontovich equations:

$$
\begin{aligned}
& {\left[\frac{\partial}{\partial t}+v \frac{\partial}{\partial x}-\frac{e}{m} E(x, t) \frac{\partial}{\partial v}\right] f(x, v, t)=0} \\
& \frac{\partial}{\partial x} E(x, t)=4 \pi e n_{0}\left\{1-\int d v E(x, v, t)\right\},
\end{aligned}
$$

where $m$, $-e$ and $n_{0}$ are the mass, charge and average density of the electron and $f(x, v, t)$ is the microscopic electron distribution function which satisfies the initial condition:

$$
f(x, v, 0)=\frac{1}{n_{0}} \sum_{j} \delta\left[x-x_{j}(0)\right] \delta\left[\dot{v}-v_{j}(0)\right]
$$

$x_{j}(0), v_{j}(0)$ being the initial position and velocity of the j-th particle respectively. Averaging eqs. (2-1) and (2-2) with respect to the initial preparation of the system, we obtain

$$
\begin{aligned}
& {\left[\frac{\partial}{\partial t}+v \frac{\partial}{\partial x}-\frac{e}{m}<E(x, t)>\frac{\partial}{\partial v}\right]\langle f(x, v, t)\rangle=\frac{e}{m} \frac{\partial}{\partial v}<E(x, t) f(x, v, t)>c} \\
& \frac{\partial}{\partial x}<E(x, t)>=4 \pi e n_{0}\left[1-\int d v<f(x, v, t)>\right],
\end{aligned}
$$

where the angular bracket means the ensemble average and 
$\langle E(x, t) f(x, v, t)\rangle_{c}=\langle E(x, t) f(x, v, t)\rangle-\langle E(x, t)\rangle\langle f(x, v, t)\rangle$.

If we adopt the vlasov approximation, eq. (2-4) is reduced to

$$
\left[\frac{\partial}{\partial t}+v \frac{\partial}{\partial x}-\frac{e}{m}\langle E(x, t)\rangle \frac{\partial}{\partial v}\right]\langle f(x, v, t)\rangle=0 .
$$

As the average state satisfying eqs. (2-4') and (2-5), we consider a situation in which there is a monochromatic stationarily traveling wave of the form,

$$
E_{0}(x, t)=E_{0:} \cos \left(k_{0:} x-\omega_{0}, t\right)
$$

eqs. (2-4') and (2-5) are then written in the wave frame (moving with wave phase velocity $v_{0}=\frac{h_{0}}{k_{0}}$ ) as follows:

$$
\begin{aligned}
& {\left[v \frac{\partial}{\partial x}-\frac{e}{m} E_{0}(x) \frac{\partial}{\partial v}\right] F(x, v)=0} \\
& \frac{\partial}{\partial x} E_{0}(x)=4 \text { men, }\left[1-\int d v F(x, v)\right] .
\end{aligned}
$$

where $E_{0}(x)$ and $F(x, v)$ are the average electrostatic field and distribution function in the wave frame.

From eqs. $(2-1),(2-2),(2-7)$ and $(2-8)$ we can obtain the equations governing the fluctuating quantitieg

$$
f^{\mu}(x, v, t)=f(x, v, t)-E(x, v), E^{\mu}(x, t)=E(x, t)-E_{0}(x)
$$

in the wave frame as follows:

$$
\begin{aligned}
& {\left[\frac{\partial}{\partial t}+v \frac{\partial}{\partial x}-\frac{e}{m} E_{D}(x) \frac{\partial}{\partial v}\right] f^{\mu}(x, v, t)=\frac{e}{m} E^{\mu}(x, t) \frac{\partial}{\partial v} F(x, v)} \\
& \frac{\partial}{\partial x} E^{\mu}(x, t)=-4 \pi e n_{0} \int d v f^{\mu}(x, v, t),
\end{aligned}
$$


where the superscript $\mu$ denotes the fluctuation from the average. In the above equation we have neglected the term $\left.\frac{e^{m}}{m}{ }^{\mu}(x, t) \frac{\partial}{\partial v} f^{\mu}(x, v, t) .7\right)+$ The general solution of eq. (2-9) consists of two parts; the particular solution of this equation and the general solution of the corresponding homogeneous equation, i.e.,

$$
\left[\frac{\partial}{\partial t}+\frac{v \partial}{\partial x}-\frac{e}{m} E_{0}(x) \frac{\partial}{\partial v}\right] f^{\mu}(x, v, t)=0
$$

Using the solution of eqs. (2-9) and (2-10) we can dexive an equation for the spectral density of the electric field in the form

$$
\left.\frac{\partial}{\partial t}\left\langle\left. E_{k}^{\mu}(t)\right|^{2}\right\rangle=2 \gamma_{k}<\left|E_{k}^{\mu}(t)\right|^{2}\right\rangle+s_{k}
$$

where $\gamma_{k}$ is the damping rate of the fluctuation of wave number $k$ and $s_{k}$ is a source term. It is this source term which gives the time rate of spontaneous emission of waves.

+ Neglect of this term is essentially equivalent to the random phase approximation in the equation for $\left\langle f^{\mu}(x, v, t) f^{\mu}\left(x^{\prime}, v^{\prime}, t^{\prime}\right)\right\rangle$. 
\$3. Solution of the Basic Equations

We first investigate the particular solution of eq. (2-9). It can be derived by the Green's function method as done by M. V. Goldman. 6) Integrating the solution over the velocity, the charge-density fluctuation $\rho^{\mu}(x, t)$ is derived as

$$
\begin{gathered}
4 \pi \rho^{\mu}(x, t)=-\int_{-\infty}^{+\infty} d x^{\prime} \int_{0}^{t} d t^{\prime} \frac{\partial}{\partial x} x\left(x, x^{\prime} ; t-t^{\prime}\right) E^{\mu}\left(x^{\prime}, t^{\prime}\right) \\
\frac{\partial}{\partial x} x\left(x, x^{\prime} z t-t^{\prime}\right) \equiv \omega_{p}^{2} \theta\left(t-t^{\prime}\right) \int_{-\infty}^{+\infty} d v^{\prime} \delta\left[x-x\left(x^{\prime}, v^{\prime} ; t-t^{\prime}\right)\right] \\
x \frac{\partial}{\partial v^{\prime}} F\left(x^{\prime}, v^{\prime}\right),
\end{gathered}
$$

where $x\left(x, x^{\prime} ; t-t^{\prime}\right)$ represents the electrostatic susceptibility of the system, $\theta\left(t-t^{\prime}\right)$ is the usual step function and $\omega_{\mathrm{p}} \equiv\left(\frac{4 m_{0} \mathrm{e}^{2}}{\mathrm{~m}}\right) 1 / 2$ (electron plasma frequency). In the above expression, $x\left(x^{\prime}, v^{\prime} ; t-t^{\prime}\right)$ and $v\left(x^{\prime}, v^{\prime} ; t-t^{\prime}\right)$ represent the orbit satisfying the following equation with the condition $x=x^{\prime}$, vev' at $t=t^{\prime}$ :

$$
\begin{aligned}
& \frac{d x}{d t}=v \\
& \frac{d v}{d t}=-\frac{e}{m} E_{0}(x) .
\end{aligned}
$$

We introduce a Fourier-Laplace component $A(k, \omega)$ of a physical quantity $A(x, t)$ défined by

$$
A(k, \omega)=\int_{-\infty}^{+\infty} d x \int_{0}^{\infty} d t e^{i \omega t-i k x} A(x, t)
$$

$A(k, w)$ being assumed to be regular in the region Imw>0. 
In this representation, eqs. $(3-1)$ and (3-2) are rewritten as

$$
\begin{aligned}
& \rho^{\mu}(k, \omega)=-i \frac{k}{4 \pi} \sum_{n=-\infty}^{+\infty} x_{n}\left(k, \omega l E^{\mu}\left(k+n k_{0}, \omega\right)\right. \\
& x_{n}(k, \omega)=-i \frac{\omega_{p}^{2}}{k} \int_{-\infty}^{+\infty} d v^{\prime} \int_{0}^{\lambda_{0}} \frac{\partial x^{\prime}}{\lambda_{0}} \int_{v}^{\infty} d t e^{i \omega t} e^{i\left(k+n k_{0}\right) x^{\prime}} \\
& x e^{-i k x\left(x^{\prime}, v^{\prime}, t\right)} \frac{j}{\partial v} F\left(x^{\prime}, v^{\prime}\right),
\end{aligned}
$$

where we have used the fact that our system is periodic in space with the period $\lambda_{0}\left(\equiv \frac{2 \pi}{\mathrm{k}_{0}}\right)$.

Next, the general solution of the homogeneous equation (2-11) can be written as

$$
f_{s}^{\mu}(x, v, t)=\frac{1}{n_{0}} \sum \delta\left[x-x_{j}(t)\right]\left[v-v_{j}(t)\right]-F(x, v)
$$

where $x_{j}(t)=x\left(x_{o_{j}}, v_{o_{j}} ; t\right), v_{j}(t)=v\left(x_{o_{j}}, v_{o_{j}} ; t\right)$ in the previous notation and suffix:s is used to indicate the solution of the homogeneous equation. The corresponding charge-density fluctuation '? becomes

$$
\begin{aligned}
\rho_{s}^{\mu}(x, t) & \equiv-n_{a} \otimes \int_{-\infty}^{+\infty} d v f_{s}^{\mu}(x, v, t) \\
& =-\theta \sum_{j}^{\Sigma} \delta\left[x-x_{j}(t)\right]-\rho_{0}(x),
\end{aligned}
$$

$\rho_{0}(x)$ being the charge density due to $F(x, v)$. The FourierLaplace component of eq. (3-9) is

$$
\rho_{s}^{\mu}(k, \omega)=-e \sum_{j} \int_{0}^{\infty} d t e^{-i k x_{j}(t)+i \omega t}-\rho_{0}\left(k_{0}\right) \delta_{k, k_{0}},(3-10)
$$


Adding this term to eq. (3-6) and using Poisson's equation, we obtain the equation for electric field $\mathrm{E}^{\mu}(\mathrm{k}, \omega)$ as follows:

$$
E^{\mu}(k, \omega)+\sum_{n=-\infty}^{+\infty} x_{n}(k, \omega) E^{\mu}\left(k+n k_{0}, \omega\right)=\frac{4 \pi i}{k} \rho_{5}^{\mu}(k, \omega) .
$$

We rewrite eq. (3-11) in terms of Fourier-Laplace components in the laboratory frame. The Fourier-Laplace component in the laboratory frame $\tilde{E}^{\mu}(k, \omega)$ is related to the component in the wave frame $E^{\mu}(k, w)$ through

$$
\widetilde{E}^{\mu}\left(k, \omega+k v_{0}\right)=E^{\mu}(k, \omega)
$$

(this can be derived using the relation between the positions in both frames.) Therefore, eq. (3-11) is written in the laboratory frame as

$$
\tilde{E}^{\mu}(k, \omega)+\sum_{n} \chi_{n}\left(k, \omega-k v_{0}\right) \tilde{E}^{\mu}\left(k+n k_{0}, \omega+n \omega,\right)=\frac{4 \pi i}{k} \tilde{\rho}_{s}^{\mu}(k, \omega) .
$$

The above equation is a matrix equation of infinite-dimension.

Let us restrict ourselves to the case of smali amplitude field E. in the sense that

$$
E_{0} \ll \sqrt{4 m_{0} T}
$$

T being the electron temperature. In this case the dispersion relation of this system is not much different from that of the homogeneous system. Therefore, we may truncate the above matrix equation, retaining only those components $\tilde{E}^{\mu}\left(k+n k_{0},(1+n a)_{0}\right)$ in eq. (3-13) whose wavenumers and frequencies nearly satisfy the $\therefore:$ dispersion relation of the homogeneous system. In the present case we have only to retain two component $\tilde{E}^{\mu}(k, \omega)$ and $\widetilde{E}^{\mu}\left(k-2 k_{0}, \omega-2 \omega_{0}\right)$, because only these can simultaneously satisfy the dispersion relation 
of the homogeneous system approximately. Then, eq. (3-11) is reduced to the coupled equations for these two components:

$$
\begin{aligned}
{\left[\begin{array}{cc}
\left\{1+x_{0}\left(k, \omega-k v_{0}\right)\right\} & x_{-2}\left(k, \omega-k v_{0}\right) \\
x_{2}\left(k-2 k_{0}, \omega-k v_{0}\right) & \left\{1+x_{0}\left(k-2 k_{0}, \omega-k v_{0}\right)\right.
\end{array}\right)\left[\begin{array}{c}
\tilde{E}^{\mu}(k, \omega) \\
\tilde{E}^{\mu}\left(k-2 k_{0}, \omega-2 \omega_{0}\right)
\end{array}\right) } \\
=\left[\begin{array}{l}
\frac{4 \pi i}{k} \tilde{\rho}_{s}^{\mu}(k, \omega) \\
\frac{4 \pi i}{k-2 k_{0}} \tilde{\rho}_{s}^{\mu}\left(k-2 k_{0}, \omega-2 \omega_{0}\right)
\end{array}\right]
\end{aligned}
$$

From the condition that the corresponding homogeneous equations have a non-trivial solution, we can obtain the dispersion equation in the present approximation as

$$
D(k, \omega)=0 \text {. }
$$

where

$$
\begin{aligned}
D(k, \omega) \equiv\left\{1+x_{0}\left(k, \omega-\mathrm{kv}_{0}\right)\right\}\left\{1+x_{0}\left(k-2 k_{0}, \omega-k_{0}\right)\right\} \\
-x_{-2}\left(k, \omega-k v_{0}\right) x_{2}\left(k-2 k_{0}, \omega-k v_{0}\right)
\end{aligned}
$$

The solution of eq. (3-16) specifies the collective oscillation of the system. Here we are not concerned with the details of the solution of $(3-16)$.

Using (3-10) one can derive the charge-density correlation

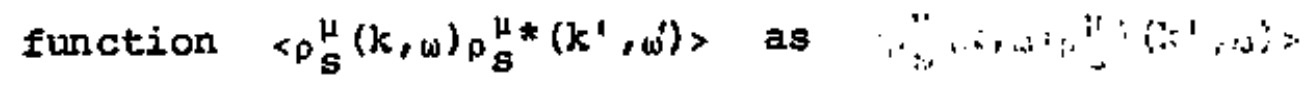

$$
\begin{aligned}
& \left\langle\rho_{s}^{\mu}(k, \omega) \rho_{s}^{\mu *}\left(k^{\prime}, \omega^{\prime}\right)\right\rangle \\
& =\int_{-\infty}^{+\infty} d x d x^{\prime} \int_{0}^{\infty} d t d t^{\prime}<\rho_{s}^{\mu}(x, t) \rho_{g}^{\mu}\left(x^{\prime}, t^{\prime}\right)>e^{-i k x+i \omega t+i k^{\prime} x^{\prime}-i \omega^{*} t} t^{\prime}
\end{aligned}
$$

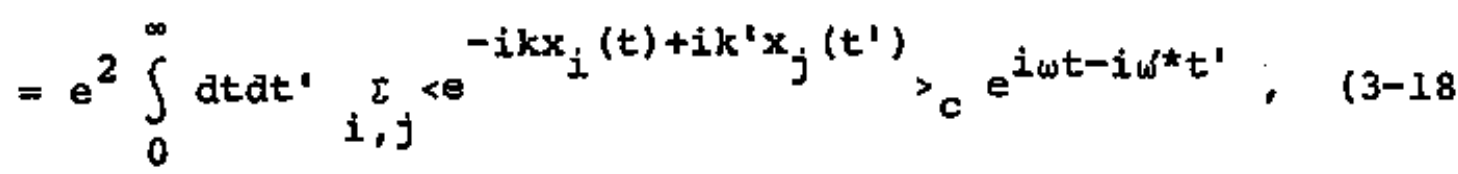


where the asterisk denotes the complex conjugate. If we neglect correlations between different particles, this reduces to $\left\langle p_{s}^{\mu}(k, w) \rho_{s}^{\mu}{ }^{\mu}\left(k^{\prime}, w\right)\right\rangle=e^{2} \int_{0}^{\infty} \operatorname{dtdt^{\prime }} \sum_{j}\left\langle e^{-i k x_{j}(t)+i k^{\prime} x_{j}\left(t^{\prime}\right)}>e^{i \omega t-i w^{\prime} t^{\prime}}\right.$ (3-19)

In the above derivation we used the relation

$$
\text { ex } \sum_{j} \delta\left[x-x_{j}(t)\right]>=-p_{0}(x)
$$




\section{Explicit Expression for Susceptibility}

In this section we wish to derive an explicit expression for the susceptibility. For this purpose we must assume explicit forms for the orbit $x\left(x^{\prime}, v^{\prime} ; t\right)$ and the average distribution function $F\left(x^{\prime}, v^{\prime}\right)$ in eq $=(3-7)$.

We first derive the orbit satisfying eqs. (3-3) and (3-4). We can put $E_{0}(x)=-E_{0} s i n k_{0} x$ without loss of generality. Then, the first integration of eq. (3-4) gives the energy conservation law in the wave frame:

$$
W \equiv \frac{I}{2} m v^{2}+\frac{e E_{0}}{k_{0}} \cos k_{0} x=\text { constant }
$$

As we can see from this law, particles can be split into two groups according to their energies; one is a group of untrapped particles whose total energies are larger than $e \mathbb{F}_{0} / k_{0}$ and the other is a group of trapped ones whose total energies are smaller than $e_{0} / k_{0}$. Although we can get the exact orbit of a particle by using an elliptic integral, we shall here be content with an approximate orbit for simplicity.

First for trapped particles, we approximate their motion by a small oscillation near the bottom of the potential trough; that is, we derive the orbit by perturbational approach with small expansion parameter $w / \frac{e_{0}}{k_{0}}(<<l)$, retaining only the lowest order term. More explicitly, we use the following approximate equation for eg. $(3-4)$

$$
\frac{d v}{d t}=-\frac{e E_{0}}{m} k_{0}\left(x-\frac{\lambda_{0}}{2}\right)
$$


where we chose a particle trapped in the trough at $x=\frac{\lambda_{0}}{2}$ as a represeritative of the trapped particles.

The solution of the above equation is of the form

$$
\begin{aligned}
& x(t)=\frac{\cdots}{\omega_{B}} \sqrt{\frac{2 W}{m}} \sin \left(\omega_{B} t+\alpha\right)+\frac{\lambda_{e}}{2} \\
& v(t)=\sqrt{\frac{2 W}{\cdot m}} \cos \left(\omega_{B} t+\alpha\right)
\end{aligned}
$$

where $\omega_{B}=\sqrt{e_{0} k_{0} / m}$ is the bounce frequency and the energy $W$ and the phase a are determined by the initial conditions. For the initial values of $x=x^{*}, v=v^{\prime}$, we have

$$
\begin{aligned}
& x(t) \equiv x_{0}^{T}\left(x^{\prime}, v^{\prime} ; t\right)=\frac{v^{\prime}}{\omega_{B}} \sin \omega_{B} t+\left(x^{\prime}-\frac{\lambda_{Q}}{2}\right) \cos \omega_{B} t+\frac{\ddot{\lambda_{0}}}{2} \\
& v(t) \equiv v_{0}^{T}\left(x^{\prime}, v^{\prime} ; t\right)=v^{\prime} \cos \omega_{B} t-\omega_{B}\left(x^{\prime}-\frac{\lambda_{0}}{2}\right) \sin \omega_{B} t .
\end{aligned}
$$

The quantities $x_{0}^{T}\left(x^{\prime}, v^{\prime} ; t\right)$ and $v_{0}^{T}\left(x^{\prime}, v^{\prime} ; t\right)$ are the expressions for $x\left(x^{\prime}, v^{\prime} ; t\right)$ and $v\left(x^{\prime}, v^{\prime} ; t\right)$ of a trapped particle in the present approximation.

For untrapped particles, we can also solve the equation perturbationally with a small expansion parameter $\frac{e E_{0}}{k_{0}} / \mathrm{h}(\ll<)$ and retain only the lowest order term. The approximate equation is

$$
\frac{\mathrm{dv}}{d t}=\frac{\mathrm{eE}_{0}}{\mathrm{~m}} \operatorname{sink_{0}}\left[\mathrm{v}_{0} t+\mathrm{x}(0)\right]
$$

and its solution is written as follows: 


$$
\begin{gathered}
x(t)=y_{1} t+\frac{e E_{0}}{m k_{0}^{2} y_{0}^{2}} \sin \left[k_{0} v_{0} t+\beta\right] . \\
v(t)=v_{0}+\frac{e E_{0}}{m k_{0} v_{0}} \cos \left[k_{0} v_{0} t+\beta\right] .
\end{gathered}
$$

where $v_{0}$ is the unperturbed velocity of a particle in the wave frame and $\beta$ is determined by the initial position of the particle. In the calculation of $x_{n}$, however, it is sufficient to use the orbit of a freely moving particle; that is,

$$
\begin{aligned}
& x(t) \equiv x_{0}^{U T}\left(x^{\prime}, v^{\prime} ; t\right)=v^{\prime} t+x^{\prime} \\
& v(t) \equiv v_{0}^{U T}\left(x^{\prime}, v^{\prime} ; t\right)=v^{\prime}
\end{aligned}
$$

$x_{0}^{U T}$ and $v_{0}^{U T}$ being the expressions for $x\left(x^{\prime}, v^{\prime} ; t\right)$ and $v\left(x^{\prime}, v^{\prime} ; t\right)$ of an untrapped particle in the present approximation.

Next, we consider the average distribution function $F(x, v)$. Detailed discussions about this function are found in the references 3) and 4). Here we use their result in the limit of small amplitude harmonic waves, i.e.

$$
F(x, v)= \begin{cases}f_{0 I}\left(\sqrt{v^{2}-\frac{2}{m} y(x)}\right) \theta\left(v^{2}-\frac{2}{m} y(x)\right) & \text { for } \\ f_{0 I}\left(-\sqrt{v^{2}-\frac{2}{m} y(x)}\right) \theta\left(v^{2}-\frac{2}{m} y(x)\right) & \text { for } v<0 \\ & +\left[f_{01}(0)-\frac{\mu}{\pi} \sqrt{\frac{2}{m} y(x)-v^{2}}\right] \theta\left(\frac{2}{m} y(x)-v^{2}\right),\end{cases}
$$

+ Error incurred by this approximation is of orderg of $\left[\mathrm{eE}_{0} /\left(k_{0} \omega\right)\right]^{2}$ and $\left[e E_{0} /\left(k_{0}, r\right)\right]^{2}$. 
where $f_{01}(v)=f_{01}(0, v)$ is the distribution function of untrapped electrons at the point $x=0$, in the wave frame, $y(x)=\frac{e E_{0}}{k_{0}}\left\{1-\cos k_{0} x\right\}$ and $\mu$ is a constant which is given by the dispersion relation:

$$
1=\frac{\omega_{p}^{2}}{k_{0}^{2}}\left[P \int_{-\infty}^{t_{\infty}} d v \frac{\dot{f}_{0 I} \cdot(v I}{v}+\mu\right] \text {. }
$$

We can see from the relation between $w$ and $y(x)$ that particles whose velocities at the point $x$ exceed $\frac{2}{\mathfrak{m}} y(x)$ are untrapped and those whose velocities at the point $x$ are smaller than $\frac{2}{\mathrm{~m}} y(x)$ are trapped.

From now on, we choose a Maxwellian distribution for $f_{01}(v)$ in the laboratory frame. That is,

$$
f_{0 I}(v)=c_{0} \exp \left[-\frac{m}{2 T}\left(v+v_{0}\right)^{2}\right],
$$

C. being a suitable normalization constant.

We are now ready to carry out the calculation of the susceptbility $x_{n}(k, w)$ given by eq. (3-7). We rewrite eq. $(3-7)$ as follows :

$$
x_{n}(k, w)=-\frac{\omega_{p}^{2}}{k} \int_{-\infty}^{+\infty} d v^{\prime} \int_{0}^{\lambda_{0}} \frac{d x^{\prime}}{\lambda_{0}} l\left(x^{\prime}, v^{\prime} ; w\right) e^{i\left(k+n k_{0}\right) x^{\prime}} \cdot \frac{\partial F\left(x^{\prime}, v^{\prime}\right)}{\partial v^{\prime}} .
$$

where we put

$$
\ell\left(x^{\prime}, v^{\prime} ;\right) \equiv i \int_{0}^{\infty} d t e^{i \omega t-i k x\left(x^{\prime}, v^{\prime} ; t\right)} .
$$

(regular at Imu>0) 
In our approximation this becomes the following form; for an untrapped particle

$$
Q^{\mathrm{UT}}\left(x^{\prime}, v^{\prime} ; \omega\right)=-\frac{e^{-i k x^{\prime}}}{\omega-k v^{\prime}}
$$

and for a trapped particle

$$
Q^{T}\left(x^{\prime}, v^{\prime} ; \omega\right)=-e^{-i \frac{k \lambda_{Q}}{2}} \sum_{n, l=-\infty}^{+\infty}(-1)^{n^{n} \frac{J_{n}\left[k \cdot\left(x^{l}+\frac{\lambda_{0}}{2}\right)\right] J_{\ell}\left(\frac{k v^{i}}{\omega_{B}}\right)}{\omega+(n-l) \omega_{B}}} .
$$

We used the expansion formula by the Bessel function in calculation of $Q^{T}$. Let us split $x_{n}(k, w)$ into two parts $x_{n}^{\text {UT }}(k, w)$ and $x_{n}^{T}(k, w)$ corresponding to contributions from two groups of electrons. That is, $x_{n}(k, w)=x_{n}^{U T}(k, \omega)+x_{n}^{T}(k, w)$, where

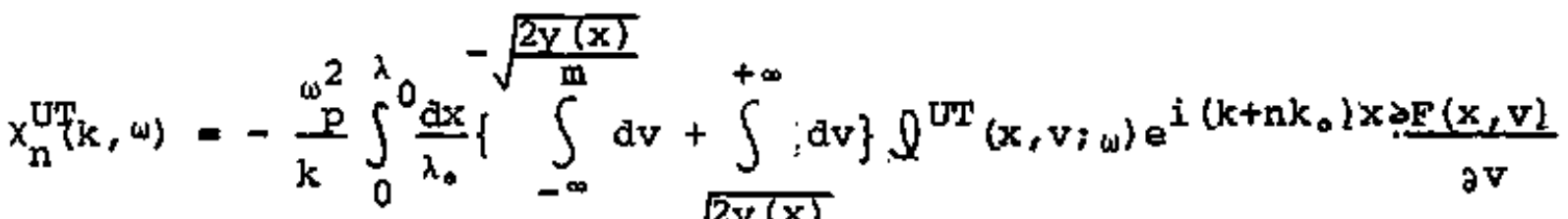

$$
\begin{aligned}
& 2 \lambda_{0}+\sqrt{\frac{2 y(x)}{\mathrm{m}}} \sqrt{\frac{2 \mathrm{yy}(x)}{\mathrm{m}}} \quad \text { (4-19) } \\
& x_{n}^{T}(k, \omega)=-\frac{\omega_{p}}{k} \int_{0} \frac{d x}{\lambda_{0}} \int_{d v} d l^{T}(x, v ; \omega) e^{i\left(k+n k_{0}\right) x} \frac{\partial^{F}(x, v)}{\partial v} \text {. } \\
& -\sqrt{\frac{2 y(x)}{m}}
\end{aligned}
$$

Substituting the explicit form for $F(x, v)$ into eqs. (4-19) and (4-20) and carrying out the integration over the velocity (see Appendix A), we obtain the following expressions correct to the first order in $y(x)$ (or $\left.E_{0}\right)$ :

$$
\begin{aligned}
x_{\dot{n}}^{U T}(k, \omega)= & -\frac{m}{T} \cdot \frac{\omega_{p}^{2}}{k^{2}} z_{0}\left(\frac{\omega}{k}\right) \delta_{n, 0} \\
& -\left(\frac{m}{T}\right)^{2} \cdot \frac{2}{k^{2}} \cdot \frac{2}{k_{0}^{2}}\left\{z_{1}\left(\frac{\omega}{k}\right)+z_{2}\left(\frac{\omega}{k}\right)\right\}\left\{\delta_{n, 0}-\frac{1}{2}\left(\delta_{n,-1}+\delta_{n, 1}\right),\right.
\end{aligned}
$$


where

$$
z_{\circ}\left(\frac{\omega}{k}\right)= \begin{cases}\int_{-\infty}^{+\infty} d v \frac{v+v_{0}}{\omega / k-v} f_{01}(v) . & \text { for } \frac{\omega}{k} \neq 0 \\ -P \int_{-\infty}^{+\infty} a v \frac{v+v_{0}}{v} f_{01}(v) & \text { for } \frac{\underline{\omega}}{k} \propto 0\end{cases}
$$

and $P$ in front of the integral sign means to take the principal value of integration at $v=0$, and

$$
\left.x_{n}^{T}(k, \omega)=(-1)^{n} \frac{\ddot{w}_{k_{0}}}{2}\right)^{2} \cdot \frac{\ddot{\mu}}{2} \sum_{s=-\infty}^{+\infty} \frac{\omega_{B}^{\omega}}{\omega+s \omega_{B}}\left\{H_{s+1, n}(k)-H_{s-1, n}(k)\right\}
$$

where

$$
\begin{aligned}
& \left.\mathrm{H}_{s, n}(k)=(-i) \mathrm{s}^{\left[B_{s, n}\right.}(k)+\frac{1}{2}\left[B_{s, n+1}(k)+B_{s, n-1}(k)\right]\right] \ldots \\
& B_{s, n}(k)=\int_{-\lambda_{0} / 2}^{\lambda_{0} / 2} \frac{d x}{\lambda_{0}} J_{s}(k x) e^{i\left(k+n k_{0}\right) x .}
\end{aligned}
$$

From the property of the Bessel function, we can verify that $\mathrm{H}_{\mathrm{s}, \mathrm{n}}\langle\mathrm{k}\rangle$ is purely real and

$$
\begin{aligned}
& \mathrm{H}_{-s, n}(k)=\mathrm{H}_{s, n}(k) \\
& B_{s, n}(k)=B_{s,-n}(k) .
\end{aligned}
$$

Note that the right-hand side of (4-22) does not contain the term corresponding to $s=0$ (see (4-23)). The dielectric constant of a homogeneous system in the laboratory frame $\varepsilon_{0}(k, w)$ is written as follows in terms of the quantities mentioned above;

$$
\varepsilon_{0}(k, \omega)=1+\chi_{\varphi}^{U T}\left(k, \omega-k v_{0}\right)
$$


but in this case the function $z_{q}\left(\frac{\omega}{k}\right)$ is given by $\int_{-\infty}^{+\infty} d v \frac{v+v_{k}}{\omega} f_{01}(v)$ always.

Equations (4-21) and (4-22) are our final results obtained for the susceptibility. 
5. Time Rate of Spontaneous Emission

We start from the coupled equation (3-15). Solving this equation for $\tilde{E}^{\mu}(k, w)$, we gat

$$
\begin{aligned}
& \tilde{E}^{\mu}(k, \omega)=\frac{4 \pi i}{D(k, \omega)}\left[\frac{\tilde{l}}{k}\left\{1+x_{0}\left(k-2 k_{0}, \omega-k v_{0}\right)\right\} \tilde{\rho}_{s}^{\mu}(k, \omega)\right. \\
&\left.-\frac{1}{k-2 k_{0}} x_{-2}\left(k, \omega-k v_{0}\right) \tilde{\rho}_{s}^{\nu}\left(k-2 k_{0} ; \omega-2 \omega_{0}\right)\right] \cdot(5-1)
\end{aligned}
$$

Using this solution, we can obtain the expression for the spectral density of electric field due to fluctuations, $U_{k}(t) \equiv<\left|E_{k}^{\mu}(t)\right|>$, as follows:

$$
\begin{aligned}
& u_{k}(t)=<\left|\int_{+} \frac{\dot{d} \omega}{2 \pi} \frac{e^{-i \omega t}}{D(k, \omega)} \tilde{E}^{\mu}(k, \omega)\right|^{2}> \\
& -(4 \pi)^{2}<! \int_{+} \frac{\alpha \omega}{2 \pi} \frac{e^{-i \omega t}}{D(k, \omega)}\left[\frac{1}{k}\left\{1+x_{0}\left(k-2 k_{0}, \omega-k V_{0}\right)\right\} \tilde{\rho}_{s}^{\mu}(k, \omega)\right. \\
& -\left.\frac{1}{k-2 k_{0}} x_{-2}\left(k, \omega-k v_{0}\right) \vec{p}_{s}^{\mu}\left(k-2 k_{0}, \omega-2 \omega_{0}\right) 1\right|^{2}{ }^{2}
\end{aligned}
$$

where the integral sign $\int_{+} \frac{d \omega}{2 \pi}$ is an abbreviation of $\int_{-\infty+i \sigma}^{+\infty+i \sigma} \frac{d \omega}{2 \pi}(\sigma>0)$.

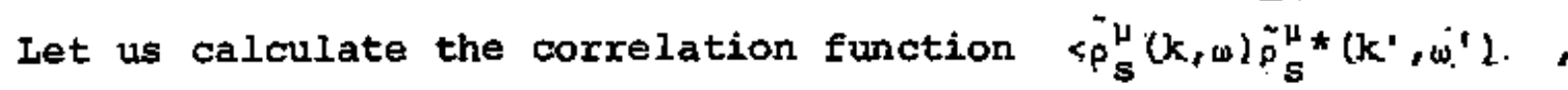
making use of eqs. $(3-19),(4-3)$ and $(4-8)$. We neglect correlations between different particles. Therefore, we can split $\left\langle\rho_{s}^{\mu} \rho_{s}^{\mu_{*}}\right.$ in to two parts as follows:

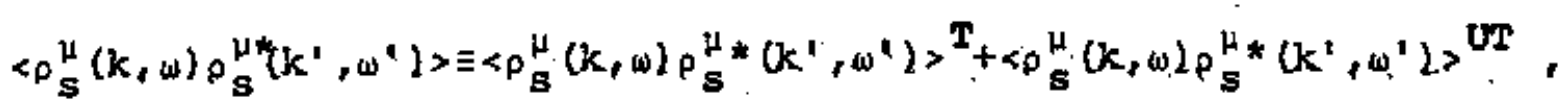

where 


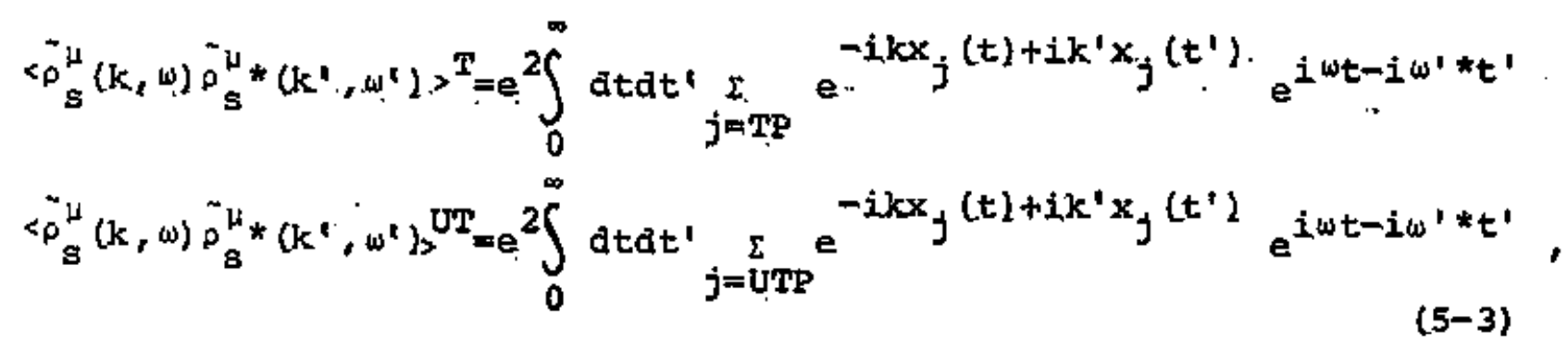
the symbols $\sum_{j=T P}$ and $\sum_{j=U T P}$ denoting the summations over the trapped and untrapped particles, respectively. After some calculations (see Appendix B), we obtain trapped-particle contribution in the wave frame

$$
\begin{aligned}
& \left\langle\rho_{s}^{\mu}(k, w) \rho_{s}^{\mu *}\left(k^{l}, w^{l}\right)\right\rangle^{T}=\sum_{j=T P} e^{2} e^{-i\left(k-k^{\prime}\right) \lambda_{o} / 2} \underset{\ell=-\infty}{\sum} J_{\ell}\left(k a_{j}\right) J_{\ell}\left(k^{\prime} a_{j}\right) \\
& \times \frac{1}{\left(\omega-\ell, \omega_{B}\right)\left(\omega \cdot{ }^{*}+\ell \omega_{B}\right)} \text {, }
\end{aligned}
$$

and for untrapped-particle contribution

$$
\begin{aligned}
& \left\langle\rho_{g}^{\mu}(k, \omega)_{s}^{\mu *\left(k^{\prime}, \omega^{\prime}\right)>\text { UT }}=\sum_{j=\mathrm{UTP}} e^{2+\infty=-\infty} \sum_{\ell}^{+\infty}\left(k b_{j}\right) J_{\ell}\left(k^{\prime} b_{j}\right)\right. \\
& \times \frac{1}{\left\{\omega-\left(k+\ell k_{0}\right) v_{0 j}\right\}\left\{\omega^{\prime} \star_{-}\left(k^{\prime}+\ell k_{0}\right) v_{0 j}\right\}} \text {. }
\end{aligned}
$$

where we put

$$
a_{j} \equiv \frac{1}{\omega_{B}} \sqrt{\frac{2 W_{j}}{m}}, b_{j} \equiv \frac{1}{k_{0}}\left(\frac{\omega_{B}}{k_{0} v_{0 j}}\right)^{2}
$$

and $w_{j}$ and $v_{\circ j}$ are the energy and unperturbed velocity of the $j-$ th particle in the wave frame, respectively. In the above derivation we assumed random distribution for the initial phases of the particles. 
Using the relations (5-4) and (5-5), we can rewrite $(5-2)$ as

$$
\begin{aligned}
& U_{k}(t)=\sum_{l=-\infty}^{+\infty} U_{k}^{T}(l ; t)+U_{k}^{U T}(t), \\
& U_{k}^{T}(l ; t) \equiv \sum_{j=T P}(4 \pi e)^{2}\left|A^{T}(j, l)\right|^{2}, \\
& U_{k}^{U T}(t) \equiv \sum_{j=U T P}(4 \pi e)^{2} \sum_{l=-\infty}^{+}\left|A^{U T}(j, l)\right|^{2},
\end{aligned}
$$

$$
\begin{aligned}
& A^{T}(j, l) \equiv \int_{+}^{d \pi} \frac{d \omega}{D(k, \omega)\left(\omega-k v_{0}-l \omega_{B}\right)}\left[\left\{1+x_{0}\left(k-2 k_{0}, \omega-k v_{0}\right)\right\} \cdot \frac{J_{l}^{-i \omega t}\left(k a_{j}\right)}{k}\right. \\
& \left.-x_{-2}\left(k, w-k v_{0}\right) \cdot \frac{J_{2}\left(\left(k-2 k_{0}\right) a_{j}\right)}{k-2 k_{0}}\right] \text {, }
\end{aligned}
$$

$$
\begin{aligned}
& A^{\mathrm{UT}}(j, \ell) \equiv \int_{+} \frac{d \omega}{2 \pi} \frac{e^{-i \omega t}}{D(k, \omega)}\left[\frac{\left\{1+x_{0}\left(k-2 k_{0}, \omega-k v_{0}\right)\right\}}{\omega-k\left(v_{0}+v_{0 j}\right)-l k_{0} v_{0 j}} \cdot \frac{J_{l}^{\prime}\left(k b_{j}\right)}{k}\right. \\
&\left.-\frac{x_{-2}\left(k, \omega-k v_{0}\right)}{\omega-k\left(v_{0}+v_{0 j}\right)-(\ell-2) k_{0} v_{0}} \cdot \frac{J_{\ell}\left(\left(k-2 k_{0}\right) b_{j}\right)}{k-2 k_{0}}\right]
\end{aligned}
$$

where we used the relation $\rho_{s}^{\mu}(k, \omega)=p_{S}^{\mu}\left(k, \omega-k v_{0}\right)$.

In the calculation of the spontaneous emission rate, it is sufficient to know a behavior of the dielectric constant near the frequencies $\omega=k \mathrm{~V}_{0}+\ell \omega_{B}$ for emissions by trapped particles and $\omega=k\left(v_{0}+v_{o j}\right)+\ell k_{0} v_{o j}$ for emissions by untrapped particles, because waves are spontaneously emitted only when a pole associated with the collective oscillation (i.e. $D(k, \omega)=0$ ) coincides with another pole associated with the individual particle motion (i.e. $\omega=k v_{0}+l \omega_{B}$ and $\omega=k\left(v_{0}+v_{o_{j}}\right)+\ell k_{0} v_{o_{j}}$ for trapped and untrapped particles respectively). This situation is essentially the same as in the 
usual treatment for a homogeneous case. ${ }^{6)}$ It should be noted that each texm, on the right-hand side of (5-7) has a definite physical meaning as the spectral density of each separate wave, since different terms in (5-7) correspond to fluctuations of different frequencies and wavenumbers, as we shall see below. tl We shall calculate $\mathrm{U}_{\mathrm{k}}^{\mathrm{T}}(l, t)$ and $\mathrm{J}_{\mathrm{k}}^{\mathrm{UT}}(t)$ in the following.

\section{(I) Trapped-particle contribution}

As seen from (4-22) and (4-23l, the susceptibility $x^{T}\left(k_{1}\left(-k_{0} v_{0}\right)\right.$ is a smooth funciton of $w$ near the frequency $w=k v_{0}$, while it becomes very large near $\omega=k v_{0}+\ell \omega_{B}(\ell \neq 0$, . This is due to the fact that the bouncing motion of trapped particles can resonate with the oscillation of a perturbed electric field of frequency $\omega=\mathrm{kV} \mathrm{V}_{0}+\ell \omega_{\mathrm{B}}(\ell \neq 0)$. Taking this resonance effect into account, we calculate $\mathrm{U}_{k}^{\mathrm{T}}(0 ; t)$ and $\mathrm{U}_{k}^{\mathrm{T}}(l ; t) \quad(l \neq 0)$ separately in the following. (i) calculation of $\mathbf{u}_{k}^{T}(0 ; t)$

We shall approximate the solution of $\mathrm{D}\left(\mathrm{k}, \mathrm{kV}, \mathrm{l}=0\right.$ by $\mathrm{k}=\mathrm{k}_{0}$. This approximation is based on the understanding that the dispersion relation (3-16) can be used to describe approximately the carrier wave itself by taking the limit $k \rightarrow k_{0}$. Comparison of the solution of $D\left(k, k v_{0}\right)=0$ with that of (4-13) shows that the error incurred by this approximation is of the order of $\mu\left(k_{0} / w_{B}\right)^{2}$ which is very small compared with unity, provided the trapped-particle density is sufficiently small compared with the total number density. Using the above approximation, we obtain after some approximate

t) See the $\delta$-functions contained in eqs. $(5-13),(5-16)$ and (5-17). 
calculations (see Appendix c)

$$
A^{T}(j, 0)=-i \frac{J_{0}\left(k_{0} a_{j}\right)}{k_{0} \omega_{0}} e^{\delta t-i \omega_{0} t}\left\{\frac{e^{-i\left(k V_{0}-\omega_{0}-i \delta\right) t}-1}{k v_{0}-\omega_{0}-i_{\delta}}\right\},
$$

where $a_{0}=\left.\frac{\partial \varepsilon_{0}}{\partial \omega_{\omega}}\right|_{\omega_{0}}$ and a small imaginary iquantity is is introduced for convenience. Inserting (5-12) into (5-8) and applying an asymptotic formula for small $\delta\left(\delta<<\omega_{0}\right), 92$ we obtain

$$
\mathrm{u}_{\mathrm{k}}^{\mathrm{T}}(0 ; t)=\sum_{j=T P} \frac{(4 \pi \mathrm{e})^{2} j_{0}^{2}\left(\mathrm{k}_{0} \mathrm{a}_{j}\right)}{\mathrm{k}_{0}^{2}} \cdot 2 \pi \mathrm{t} \cdot \delta\left(\mathrm{kV} \mathrm{v}_{0}-\omega_{0}\right)
$$

(ii) Calculation of $\mathrm{v}_{\mathrm{k}}^{\mathrm{T}}(l ; t) \quad(l \neq 0)$

In the calculation, the following approximation will be used for $D(k, w)$;

$$
\begin{aligned}
D(k, w)= & \varepsilon_{0}(k, w) \varepsilon_{0}\left(k-2 k_{0}, w-2 \omega\right) \\
& +\varepsilon_{0}(k, w) x_{0}^{T}\left(k-2 k_{0}, w-k v_{0}\right)+\varepsilon_{0}\left(k-2 k_{0}, \omega-2 w_{0}\right) x_{0}^{T}\left(k, w-k v_{0}\right)
\end{aligned}
$$

In this approximation the term $\left[x_{0}^{T}\left(k, \omega-k V_{0}\right) x_{0}^{T}\left(k-2 k_{0}, \omega-k V_{0}\right]\right.$ $\left.-x_{-2}^{T}\left(k, \omega-k v_{0}\right) x_{2}^{T}\left(k-2 k_{0}, \omega-k v_{0}\right)\right)$ is neglected (see $\left.(3-17)\right)$, since this term is of order $\left(\bar{v} / v_{t}\right)^{2} \exp \left[-2 v_{0}^{2} / \bar{v}^{2}\right]$, where $\bar{v}^{2}=T / 2 m$ and $v_{t}=\omega_{B} / k_{0}$. Near the frequency satisfying $\omega=k v_{0}+l \omega_{B}(l \neq 0)$, the texm corresponding to $s=-\ell$ in the expression for $x_{n}^{T}\left(k, \omega-k V_{0}\right)$ (see (4-22)) becomes very large, so that $D(k, w)$ can approximately be written as

$$
\begin{aligned}
D(k, \omega)=\frac{\omega_{B}}{\omega-k V_{0}-\ell \omega_{B}} & {\left[\varepsilon _ { 0 } ( k , \omega ) \left\{H_{-\ell+1,0}\left(k-2 k_{0} l-H_{-\ell-1,0}\left(k-2 k_{0}\right)\right\}\right.\right.} \\
& +\epsilon_{0}\left(k-2 k_{0}, \omega-2 \omega_{0}\left[\left\{H_{-\ell+1,0}(k)-H_{-\ell-1,0}(k)\right\}\right] .\right.
\end{aligned}
$$


Similar approximations can be used for the numerators of the integrand in (5-10). After some calculations (see Appendix $\mathrm{C}$, we get

$$
\begin{aligned}
& \left.A^{T}(j, l)=\frac{(-i\}}{k_{a_{k}}\{1+h(l)\}}\left\{J_{\ell}\left(k_{j}\right)+\frac{k}{2 k_{0}-k} g(l) J_{\ell}\left(l k-2 k_{0}\right) a_{j}\right)\right\} \\
& \times e^{\gamma_{k}(l) t-i \omega_{k}(l) t} \cdot\left\{\frac{j\left(k v_{0}+l \omega_{B}-\omega_{k}(l)-i \gamma_{j}(l)\right) t}{k v_{0}+l \omega_{B}-\omega_{k}(l)-i \gamma_{k}(l)}\right],
\end{aligned}
$$

where

$$
\begin{aligned}
& \omega_{k}(l) \equiv \frac{\omega_{k}^{o+h(l)}\left\{2 \omega_{0}+\omega_{k}^{0}-2 k_{0}^{0}\right\}}{1+h(l)} \\
& \gamma_{k}(\ell) \equiv \frac{r_{k}^{o}+h(\ell) \gamma_{k}^{o}-2 k_{0}}{1+h(\ell)} \\
& g(l) \equiv \frac{\mathrm{H}_{-l+1,-2}(\mathrm{k})-\mathrm{H}_{-\ell-1,-2}(\mathrm{k})}{\mathrm{H}_{-\ell+1,0}\left(\mathrm{k}-2 \mathrm{k}_{0}\right)-\mathrm{H}_{-\ell-1,0}\left(\mathrm{k}-2 \mathrm{k}_{0}\right)} \\
& h(l) \equiv \frac{\alpha_{k}-2 k_{0}}{\alpha_{k}} \cdot \frac{\mathrm{H}_{-\ell}+1,0}{\mathrm{H}_{-\ell+1,0}\left(\mathrm{k}-2 \mathrm{k}_{0}\right)-\mathrm{H}_{-\ell-1,0}\left(\mathrm{k}-2 \mathrm{k}_{0}\right)}
\end{aligned}
$$

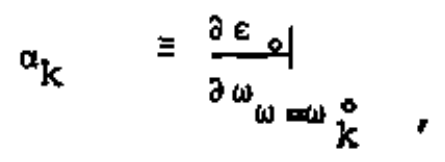

$\omega_{k}^{0}$ and $\gamma_{k}^{\circ}$ being the real and imaginary parts of the zero of $E_{0}(k, w)$, respectively. Inserting (5-14) into (5-8) and applying the same asymptotic formula for $\operatorname{small} \gamma_{k}^{(l)}\left(\gamma_{k}(l)<<\omega_{k}(l) l\right.$ as in case (i), we obtain

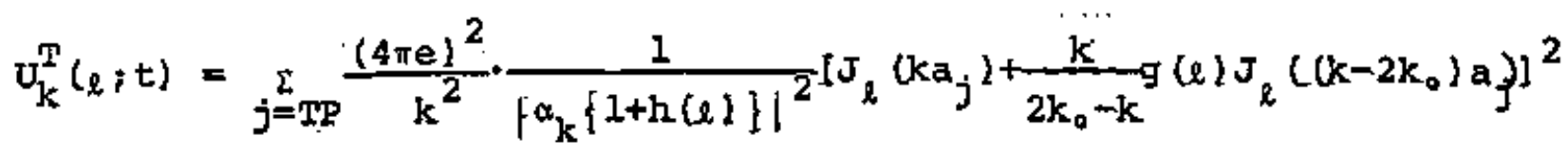

$$
\begin{aligned}
& \frac{\pi}{\gamma_{k}(l)}\left\{e^{2 \gamma_{k}(l) t}-1\right\} \delta\left[k v_{0}+\ell \omega_{B}-w_{k}(l)\right] \text {. }
\end{aligned}
$$


(II). Untrapped-particle contribution

We confine our attention to the emission by those particles whose velocities in the wave frame satisfy the condition:

$$
\left.v_{0 j}^{2} \gg . \stackrel{\omega}{B}_{k_{0}}^{2}\right)^{2} \text {. }
$$

We shall neglect the emission by other particles for simplicity. By the same reason as for the trapped-particle case, we replace the dielectric function by an approximate expression which is correct in the region of $w$ near $\left[k\left(v_{0}+v_{0 j}\right)+k_{0} v_{0 j}\right]$. We then find that the first term in the square bracket of the integrand in (5-11) gives the dominant contribution to $A^{U T}(j, l)$ (see Appenaix c). The final result is given by

$$
\begin{aligned}
& U_{k}^{U T}(t)=\sum_{j=U T P}^{\sum} \frac{(4 \pi e)^{2}}{k^{2}\left|\alpha_{k}\right|^{2}} \cdot \frac{\pi}{Y_{k}^{0}}\left\{e^{2 \gamma_{k}^{0}}-1\right\} \underbrace{+\infty}_{\ell=-\infty} J_{\ell}^{2}\left(k b_{j}\right) \\
& x \delta\left[k\left[v_{0}+v_{0 j}\right]+2 k_{0} v_{0 j}-\omega_{k}^{0}\right] \text {. }
\end{aligned}
$$

We now derive a kinetic wave equation for the spectral density by differentiating eqs. (5-13), (5-16) and (5-13) with time. That is,

$$
\begin{aligned}
& \frac{\partial}{\partial t} U_{k}^{T}(0 ; t)=2 \pi \sum_{j=T P} \frac{(4 \pi e)^{2}}{k^{2}} \cdot \frac{J_{0}^{2}\left(k_{0} a_{j}\right)}{\left|a_{0}\right|^{2}} \delta\left(k v_{0}-w_{0}\right) \\
& \left\{\frac{\partial}{\partial t}-2 \gamma_{k}(\ell)\right\} \mathrm{U}_{\mathrm{K}}^{\mathrm{T}}(\ell ; t)=2 \pi \underset{j=\mathrm{TP}}{\sum_{\mathrm{T}}} \frac{(4 \pi \mathrm{e})^{2}}{\mathrm{k}^{2}} \cdot \frac{1}{\left|\alpha_{k}\{I+h(l)\}\right|^{2}} \\
& \left.\times\left[J_{\ell}\left(k a_{j}\right)+\frac{k}{2 k_{0}-k}-g(l) J_{\ell}\left(k-2 k_{0}\right) a_{j}\right)\right]^{2} \delta\left[k v_{0}+\ell \omega_{B}-\omega_{k}(l)\right] \\
& (e+0)
\end{aligned}
$$

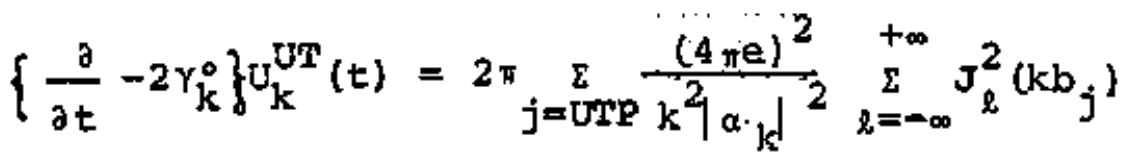

$$
\begin{aligned}
& x \delta\left[k\left(v_{0}+v_{o j} l+\ell k_{0} v_{0 j}-w_{k}^{0}\right]\right)
\end{aligned}
$$


These equations are of the form of eq. (2-12). As pointed out there; the right-hand sides give the time rates of spontaneous emission for the corresponding fluctuations.

Integrating the right-hand sides over $k$ and summing up over $\ell$. for the trapped-particle case, we obtain the time rates of total emissions by the trapped and untrapped particles (to be denoted by $I^{T}$ and $I^{U T}$, respectivelyl. They are

$$
\begin{aligned}
& I=\sum_{j=T P} \int d x\left[\frac{(4 \pi e)^{2}}{k_{0}^{2}} \cdot \frac{J_{0}^{2}\left(k_{0} a_{j}\right)}{\left|\alpha_{0}\right|^{2}} \delta\left(k v_{0}-\omega_{0}\right)\right. \\
& +\sum_{\ell \neq 0} \frac{(4 \pi e)^{2}}{k^{2}} \cdot \frac{1}{\mid a_{k}\left(1+h(l)||^{2}\right.}\left(J_{\ell}\left(k a_{j}\right)+\frac{j}{2 k_{0}-k} g(l) J_{\ell}\left(\left(k-2 k_{0}\right) a_{j}\right)\right)^{2} \\
& \left.\times \delta\left(k v_{0}+\ell \omega_{B}-\omega_{k}(l)\right)\right]
\end{aligned}
$$

and

$$
I^{U T}=\sum_{j=U T P} \int a k-\frac{(4 \pi e)^{2}}{k^{2}\left|a_{k}\right|^{2}} \underset{\ell=-\infty}{+\infty} J_{\ell}^{2}\left(k b_{j}\right) \&\left[k\left(v_{o}+v_{o_{j}}\right)+\ell k_{0} v_{o_{j}}-w_{k}^{0}\right] \text {. }
$$

Note that these results can be used not only for a stable case but also for a weakly unstable case, because we assumed only the smallness of the imaginary part of the frequency compared to the real part. 9) 
56. Discussions

Let us discuss the results obtained in 55 by investigating the emission rate and the resonance condition (the condition which determines the frequency and wavenumber of the wave eraitted).

We first consider the emission by trapped particles. In the integrand of (5-21), the first term gives the emission of the carrier wave itself. Its emission rate is the same as was obtained in our previous paper, ${ }^{3}$ ) and can become quite large, since all the trapped particles take part in this emission. Strictly speaking, the presence of this emission produces a difflculty in the present formulation, since the carrier-wave amplitude continuously increases due to this emission. Note that we are considering a B.G.K. state as the unperturbed state, so that the carrier wave does not suffer any Landau damping. In reality, however, the spontaneous emission produces a modification of the average distribution and this modification in turn affects the stationary character of the B.G.K. state. Thus for a complete discussion of the stationary state, it appears necessary to consider a coupled equation for the spectral wave energy and the average distribution, as done in the quasilinear theory.

The resonance conditions in the terms with $\ell=0$ in $(5-21)$ indicate emission of those sideband waves whose wavenumbers and frequencies are given by the relations $k v_{0}+2 \omega_{B}=\omega_{k}(\ell)$ and $\omega=\omega_{k}(\ell)$. These sidebands constitute a discrete spectrum with the fxequency separation of order " ${ }_{B}$ around the carrier-wave frequency $\omega_{0}$. A similar kind of sideband enission was also obtained in our previous work, but our new result includes two important 
modifications. First, the frequency yersus wayenumber relation is now given by $w_{\omega_{k}}(l)$, but not by the Bohm-Gross frequency; this modification arises from that of the effective dielectric function from $\varepsilon_{0}(k, w)$ to $\left\{\varepsilon_{0}(k, \omega)+h(l) \varepsilon_{0}\left(k-2 k_{0}, \omega-2 \omega_{0}\right)\right.$, which is a certain average of the dielectric functions for the two waves, $(k, w)$ and $\left(k-2 k_{0}, \omega-2 \omega_{0}\right)$, in the homogeneous system. Second $2_{y}$, the emission rate is modified by two effects, one due to the above modification of the dielectric function and the other due to the presence of two charge fluctuations, $\tilde{\rho}_{\mathrm{s}}^{\mu}(k, w)$ and $\tilde{\rho}_{\mathrm{s}}^{\mu}\left(k-2 k_{0}, w-2 \omega_{0}\right)$, which contribute to the fluctuating field $\tilde{\mathrm{E}}^{\mu}(\mathrm{k}, \omega)$. Either of these two effects, when taken independently, is very large, but their effects tend to cancel each other, so that their resultant effect is not quite substantial. An estimate for the case of $l= \pm 1$ and $k_{0} a_{j}<1$ shows that the new emission rate is one order of magnitude less than the result obtained in our previous work.

Equation (5-22), obtained for the emission by untrapped particles, indicates that an untrapped particle also emits many waves including the one which satisfies the usual Cerenkov condition, i.e. the term with $l=0$ in the sum. The terms with $l=0$ correspond to the scattering of the carrier wave by the particle. These results for the emission by untrapped particles are the same as those obtained in our previous work.

To sumarize, one can state that the effect of the modification of the dielectric property of the plasma appears mainly on the emission by the trapped particles. This is naturally untderstood since the effects mentioned above come mainly from the trapped-particle susceptibility $x^{T}$. 
In the present theory, we have ignored the correlation among different particles: In reality, the motions of those trapped particles which are localized in the same potential trough of the carrier wave can become strongly correlated to each other. This may cause a coherence effect on the spontaneous emission, namely the emission rate may become proportional to the square of the : trapped particle density in a given potential trough. In addition to this, another type of coherence effect can be expected due to a periodic array of the trapped particles. Namely, waves emitted in different potential troughs can be superposed in phase. These coherence effects can be expected only for the emission of the carrier wave itself. 


\section{Acknowledgments}

The author wishes to thank Professor K. N1shikawa for his continual guldance and his kinoness in reading and improving the original manuscript. Thanks are also due to Dr. T. Sato, Messrs. K. Mima and H. Tomita for some valuable discussions. 
Appendjx A

- Integration over velocity in $x_{n}$ -

Substituting the explicit form of eq. (4-12) for $F(x, v)$ Into eqs. (4-19) and (4-20), we obtain

$$
\begin{aligned}
& x_{n}^{U T}(k, w)
\end{aligned}
$$

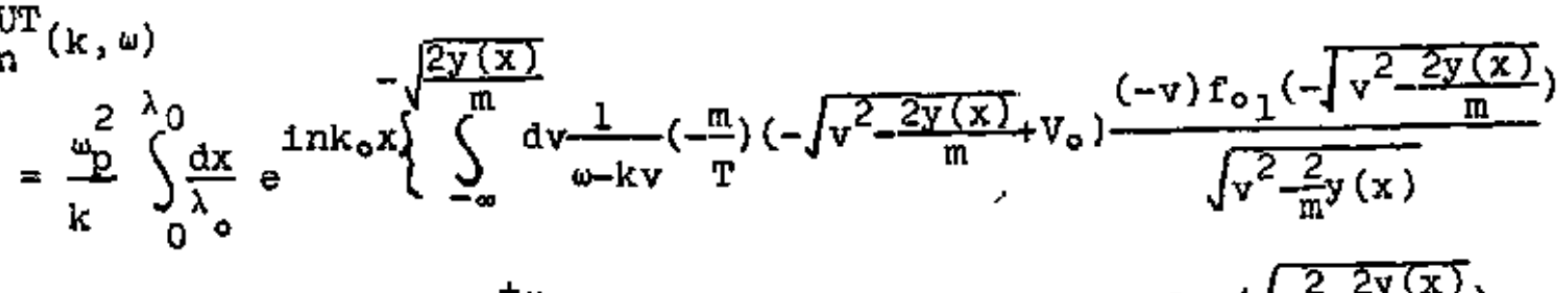

$$
\begin{aligned}
& \left.+\int_{\sqrt{\frac{2 y(x)}{m}}}^{+\infty} d v \frac{1}{\omega-k v}\left(-\frac{m}{\mathrm{t}}\right)\left(\sqrt{v^{2}-\frac{2 v(x)}{m}}+v_{0}\right) \frac{v f_{0_{1}}\left(\sqrt{v^{2}-\frac{2 y(x)}{m}}\right)}{\sqrt{v^{2}-\frac{2}{m} y(x)}}\right\} \\
& x_{n}^{T}(k, \omega)=\frac{\omega_{p}^{2}}{k} e^{-j \frac{k \lambda_{0}}{2}} \sum_{s, l=-\infty}^{+\infty}(-1)^{s} \frac{1}{\omega+(s-l) \omega_{B}} \int_{0}^{\lambda_{0}} \int_{0} \frac{\partial x}{\lambda_{0}}\left[k\left(x-\frac{\lambda}{2}\right)\right] e^{1\left(k+n k_{0}\right) x} \\
& +\sqrt{\frac{2 y(x)}{m}} \\
& \times \frac{\mu}{\pi} \int_{-\sqrt{\frac{2 \bar{y}(x)}{m}}} d v J_{\ell}\left(\frac{k v}{\omega_{B}}\right) \cdot \frac{v}{\sqrt{\frac{2}{m} y(x)-v^{2}}} .
\end{aligned}
$$

(1) Calculation in $x_{n}^{T}$ :

$$
\begin{aligned}
& A_{\ell}(x) \equiv \frac{\mu}{\pi} \frac{\sqrt{\frac{2 y(x)}{m}}}{-\sqrt{\frac{2 y(x)}{m}}} d v J_{\ell}\left(\frac{k v}{\omega_{B}}\right) \frac{v}{\sqrt{\frac{2}{m} y(x)-v^{2}}}
\end{aligned}
$$

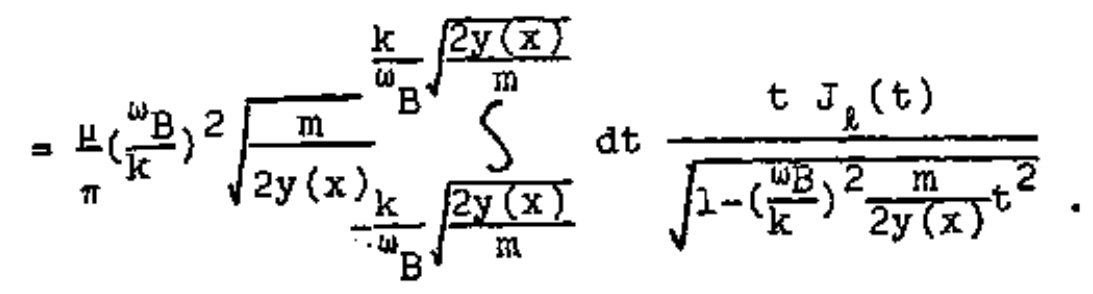


Making use of properties of the Bessel function, we obtain

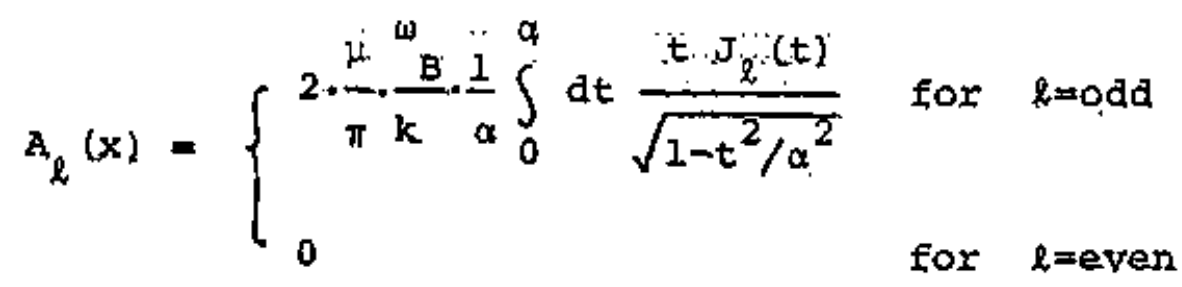

and

$$
A_{-\ell}(x)=(-I)^{\ell} A_{\ell}(x),
$$

where we put $a \frac{k}{\omega_{B}} \sqrt{2 y(x) / m}$

$$
\left[\alpha^{2}=\frac{k^{2}}{\omega_{B}^{2}} \cdot \frac{2}{m} \cdot \frac{e E_{0}}{k_{0}}\left(1-\cos k_{0} x\right)=4 \cdot\left(\frac{k_{k}^{\prime}}{k_{0}}\right)^{2} \sin ^{2}\left(\frac{k_{o} x_{1}}{2} l\right] .\right.
$$

Expanding $J_{\ell}(t)$ in power series of $t$ and integrating over $t$, we obtain

$$
\int_{0}^{a} d t \frac{t J_{2 \ell+1}(t)}{\sqrt{1-t^{2} / \alpha^{2}}}=\frac{a^{2 \ell+3}}{2^{2 \ell+1}} \sum \frac{(-1)^{n}}{n !(2 \ell+n+1) !} \cdot \underbrace{}_{2})^{2 n} \frac{\{2(\ell+n+1)-1\} !}{\{2(\ell+n+1)\} ! !} \cdot \frac{\pi}{2} .
$$

Then, substituting this result into the expression of $x_{n}^{T}$ and carrying out the $x$-integration, we find that contributions from higher-order terms in $\alpha$ become smaller (see the factor $\sin ^{2}\left(\frac{k_{g} x}{2}\right.$ ) in eq. (A6)). So we retain only the lowest-order terms in $\alpha$ and obtain eq. $(4-25)$.

(ii) Calculation in $x_{n}^{U T}$ : In case that $Y(x) / T<51$, we can expand the functions in the integrand of eq. (Al) as follows: 


$$
\begin{gathered}
\pm \sqrt{v^{2}-\frac{2}{m} y(x)}=v\left\{1-\frac{y(x)}{m v^{2}}+\cdots\right\} \\
\pm 1 \sqrt{v^{2}-\frac{2}{m^{2}}(x)}=\frac{1}{v}\left\{1+\frac{y(x)}{m v^{2}}+\cdots\right\},
\end{gathered}
$$

and

$$
f_{01}\left( \pm \sqrt{v^{2}-\frac{2}{m} y(x)}\right)=f_{01}(v)\left\{1+\frac{v+v_{0}}{v} \cdot \frac{y(x)}{T}+\cdots\right\}
$$

Substituting these expansions into the corresponding factors and considering the fact $y(x) / T<<1$, we obtain eq = (4-21). 


\section{Appendix B}

- Calculation of Charge-Density correlation Functions -

We can calculate these quantities by gubstituting the explicit orbits of eqs. $(4-3)$ and $(4-8)$ into eq. $(3-19)$.

(i) Trappedmparticle case

Using eq. (4-3), we get

Then,

$$
\begin{aligned}
e^{-i k x_{j}(t)} & =e^{i k \frac{\lambda l}{2}} e^{-i k a_{j} \sin \left(\omega_{B} t+\alpha\right)} \\
& =e^{-i k \frac{\lambda_{l}}{2}} \sum_{l} J_{\ell}\left(k a_{j}\right) e^{-i l \omega_{B} t-i l \alpha} .
\end{aligned}
$$

$$
\begin{aligned}
\left\langle e^{-i k x_{j}(t)+i k^{2} x_{j}\left(t^{\prime}\right)}\right\rangle=e^{-i\left(k-k^{\prime}\right) \frac{\lambda_{0}}{2}} \sum_{l, s} J_{l}\left(k a_{j}\right) J_{s}\left(k^{\prime} a_{j}\right) \\
x e^{-i\left(l \omega_{B} t-s \omega_{B} t^{\prime}\right)}<e^{-i(l-s) \alpha_{j}} .
\end{aligned}
$$

Assuming no initial-phase correlation, we find that this reduces to

$$
\begin{aligned}
& \left\langle e^{-i k x_{j}(t)+i k^{\prime} x_{j}\left(t^{\prime}\right)}>\right. \\
& \quad=e^{-i\left(k-k^{\prime}\right) \frac{\lambda_{0}}{2}} \sum_{\ell} J_{\ell}\left(k a_{j}\right) J_{\ell}\left(k^{\prime} a_{j}\right) e^{-i \ell \omega_{B}\left(t-t^{\prime}\right)} .
\end{aligned}
$$

Then, we obtain

$$
\begin{aligned}
& \int_{0}^{\infty} \operatorname{atdt^{\prime }}<e^{-i k x_{j}(t)+i k^{\prime} x_{j}\left(t^{\prime}\right)}>e^{i \omega t-i \tilde{\omega} * t^{\prime}} \\
& -e^{-i\left(k-k^{\prime}\right) \frac{\lambda_{l}}{2}} \sum_{\ell} J_{\ell}\left(k a_{j}\right) J_{\ell}\left(k^{\prime} a_{j}\right) \frac{1}{\left(\omega-\ell \omega_{B}\right)\left(\omega^{*}-\ell \omega_{B}\right)} \\
& (\operatorname{Im} \omega, \tilde{\omega}>0)
\end{aligned}
$$


(ii) Untrapped-particle case

Using the orbit of ex. (4-8), we get under the assumption of no initial-phase correlation,

$$
\begin{aligned}
& \left\langle e^{-i k x_{j}(t)+i k^{\prime} x_{j}\left(t^{\prime}\right)}\right\rangle \\
& \quad=e^{-i\left(k+l k_{0}\right) v_{0} t+i\left(k^{i}+l k_{0}\right) v_{0} t^{\prime}} \sum_{l} J_{l}\left(k b_{j}\right) J_{l}\left(k^{\prime} b_{j}\right)
\end{aligned}
$$

Then, we obtain

$$
\begin{aligned}
& \int_{0}^{\infty} \operatorname{dtdt}^{\prime}<e^{-i k x_{j}(t)+i k k^{\prime} x_{j}\left(t^{\prime}\right)}>e^{i \omega t-i \omega^{*} t^{\prime}} \\
& =\sum_{\ell} J_{\ell}\left(k b_{j}\right) J_{\ell}\left(k^{\prime} b_{j}\right) \frac{1}{\left\{\omega-\left(k+l k_{0}\right) v_{0 j}\right\}\left\{\omega^{*}-\left(k^{\prime}+l k_{0}\right) v_{0 j}\right\}} . \\
& \text { (Imw, } \vec{\omega}>0)
\end{aligned}
$$




\section{Appendix C}

- Calculation of $\mathrm{A}^{\mathrm{T}}$ and $\mathrm{A}^{\mathrm{UT}}$ -

(i) $A^{T}(j, 0)$

When $k \sim k_{0}$ and $\omega_{n} k V_{0} \sim \omega_{0}$, we can show the following relations making use of eqs. (4-21) and (4-22):

$$
\begin{aligned}
& x_{0}\left(k, \omega-k v_{0}\right) \simeq x_{0}\left(k-2 k_{0}, \omega-k V_{0}\right) \\
& x_{-2}^{T}\left(k, \omega-k v_{0}\right) \simeq x_{2}^{T}\left(k-2 k_{0}, \omega-k v_{0}\right)
\end{aligned}
$$

and therefore

$$
\begin{array}{r}
D(k, \omega) \simeq\left\{1+\chi_{0}\left(k, \omega-k V_{0}\right)+x_{-2}^{T}\left(k, \omega-k V_{0}\right)\right\}\left\{1+\chi_{0}\left(k-2 k_{0}, \omega-k V_{0}\right)\right. \\
\left.-x_{2}^{T}\left(k-2 k_{0}, \omega-k V_{0}\right)\right\} .
\end{array}
$$

Using the above relation, we obtain

$$
A^{T}(j, 0) \simeq \frac{J_{0}\left(k_{0} a_{j}\right)}{k_{0}} \int_{+} \frac{d \omega}{2 \pi} \frac{e^{-i \omega t}}{\left(\omega-k v_{0}\right)\left\{1+\chi_{0}\left(k, \omega-k v_{0}\right)-\chi_{-2}^{T}\left(k, \omega-k v_{0}\right)\right\}} .
$$

Near $\omega=\omega_{0}$ the function $\left\{1+x_{0}\left(k, \omega-k v_{0}\right)-\chi_{-2}^{T}\left(k, \omega-k v_{0}\right\}\right.$ can be approximated as follows;

$$
\left[1+\chi_{0}\left(k, \omega-k v_{0}\right)-\chi_{-2}^{T}\left(k, \omega-k v_{0}\right)\right]=\alpha_{0}\left\{\omega-\omega_{0}-i \delta\right\},
$$

where the coefficient $\alpha_{0}$ can approximately be written as $\left.\alpha_{0} \approx \frac{\partial E_{0}}{\partial \omega_{\omega}}\right|_{\omega=\omega_{*}}$. Substituting (C2) into (C1) and closing the integration path in the lower-half plane, we obtain eq. (5-12). 
(ii). $A^{T}(j, l)$

Using the approximate forms for the integrand of eq. $(5-10)$, we obtain

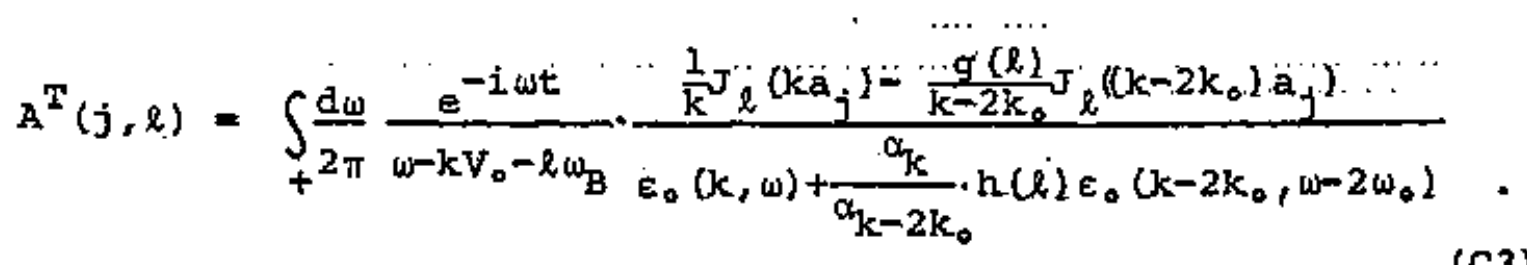

We make the resonance approximation for $\varepsilon_{0}$;

$$
\begin{aligned}
& \varepsilon_{0}(k, \omega)+\frac{\alpha_{k}}{\alpha_{k-2 k_{0}}} h(l) \varepsilon_{0}\left(k-2 k_{0}, \omega-2 \omega_{0}\right) \\
& \quad=\alpha_{k}\left\{\omega-\omega_{k}^{0}-i \gamma_{k}^{0}\right\}+\alpha_{k} h(l)\left\{\omega-2 \omega_{0}-\omega_{k-2 k_{0}}^{0}-i \gamma_{k-2 k_{0}}\right\} \\
& =\alpha_{k}\{1+h(l)\}\left\{\omega-\omega_{k}(l)-i \gamma_{k}(l)\right\} .
\end{aligned}
$$

Substituting this relation into (C3) and closing the contour in the lower-half plane, we get eq. (5-14).

$$
\text { (iii) } \mathrm{A}^{\mathrm{UT}}(j, \ell)
$$

The susceptibility $X_{n}^{T}$ can be estimated as follows at the point $\omega=k\left(V_{0}+v_{0} j_{\omega_{0}}\right)+\ell k_{0} v_{0 j}$ for those particles which satisfy the condition $v_{e}^{2}>>\left(\frac{\omega_{B}}{k_{0}}\right)^{2}$ : For $l \neq-1$

$$
\begin{aligned}
& x_{n}^{T}\left[k, k v_{0 j}+l k_{0} v_{0_{j}}\right]=(-1)^{n} \sum_{s} \frac{2 \omega_{B}^{2} \cdots}{\left\{\left(k+l k_{0}\right) v_{0 j}+s \omega_{B}\right\}^{2}-\omega_{B}^{2}} H_{S, n}(k) \\
& \left.\sim \frac{1}{(l+1)^{2}} \cdot \frac{\dot{\omega}_{p}^{2}}{\left(k_{0} v_{0 j}\right)^{2}} \cdot \frac{\ddot{\omega}_{\mathrm{B}}}{\mathrm{k}_{0}}\right)^{2} \\
& \sim \frac{I}{(l+1)^{2}} \frac{\left|\mathrm{n}_{\mathrm{B}}\right|}{\mathrm{n}_{0}} \ll 1
\end{aligned}
$$


and for $l=-1 \quad\left(k \neq k_{,}\right)$

$$
\begin{aligned}
x_{n}^{\mathrm{T}}\left[k_{i}\left(k-k_{0}\right) v_{o_{j}}\right] & =(-1)^{\mathrm{n}} \frac{1}{\left(k-\mathrm{k}_{0}\right) v_{o j}}\left\{\mathrm{H}_{1, n}(k)-\mathrm{H}_{-1, n}(k)\right\} \\
& =0, .
\end{aligned}
$$

where

$$
\begin{aligned}
& n_{T} \equiv n_{0} \int_{-\lambda_{0} / 2}^{\lambda_{0} / 2} \frac{d x}{\lambda_{0}} \int_{-\sqrt{2 y(x) / m}}^{\sqrt{2 Y(x) / m}} d v F(x, v) \text { (trapped-particle number) } \\
&=n_{0}\left\{\frac{8 f_{0}(0)}{\pi}-\mu \cdot \frac{\omega_{B}}{k_{0}}\right\} \frac{\omega_{B}}{k_{0}} \\
& n_{B} \equiv \mu \cdot \frac{\left(\frac{\omega_{B}}{k_{0}}\right)}{\pi} \quad \text { (effective-oscillator number) } \\
&=\frac{8}{\pi} f_{0}(0) \cdot \frac{\omega_{B}}{k_{0}}-n_{T} / n_{0} \\
&\left(n_{B}\right. \text { can be negative) }
\end{aligned}
$$

and we used the following evaluation;

$$
\begin{aligned}
\left(k_{0} v_{\circ j}\right)^{2} & =k_{0}^{2}\left(v_{0}-\bar{v}\right)^{2} \\
& =\omega_{0}^{2}\left(1-k_{0} \lambda_{0}\right)^{2} \\
& \sim \omega_{p}^{2} .
\end{aligned}
$$

We, therefore, neglect $x_{n}^{T}$ as being small compared to $\varepsilon_{0}$ in this case. 
References

1) B. B. Kadomtsev: Plasma Turbulence (Academic Press Inc., Iondon and New York, 1965), Chap. 2.

2) Y. Katayama: J. Phys. Soc. Japan 31 (1971) 959; KakuyugoKenkyu, Circular in Japanese, 26 (Aug. 1971) 320.

3) I. B. Bernstein, J. M. Greene and M. D. Kruskal: Phys. Rev. 108 (1957) 546.

4) M. Kito and I. Kaji: J. Phys. Soc. Japan 19 (1964) 555.

5) See, for instance, W. L. Kruer, J. M. Dawson and R. N. Sudan: Phys. Rev. Letters 23 (1969) 838.

6) K. Nishikawa and Y. Osaka: Prog. Theor. Phys. 33 (1965) 402.

7) Yu. L. Klimontovich: The Statistical Theory of Non-Equilibrium Processes in a Plasma (Pergamon Press, 1967), Chap. 2.

8) M. V. Goldman: Phys of Fluids 13 (1970) 1281.

9) K. Nishikawa: Prog. Theor. Phys. 36 (1966) 193. 


\section{Figure Caption}

F18. 1 The curve of $w=\widetilde{\omega}_{k}(l)$ and $w=k V_{0}+l w_{B}$. The intersecting point $\left(\omega_{\ell}(k), k_{l}\right)$ gives the frequency and wave number of the wave which aatisfies the resonance condition $k_{0}+\ell w_{B}=$ $\widetilde{\omega}_{k}(l)$. 


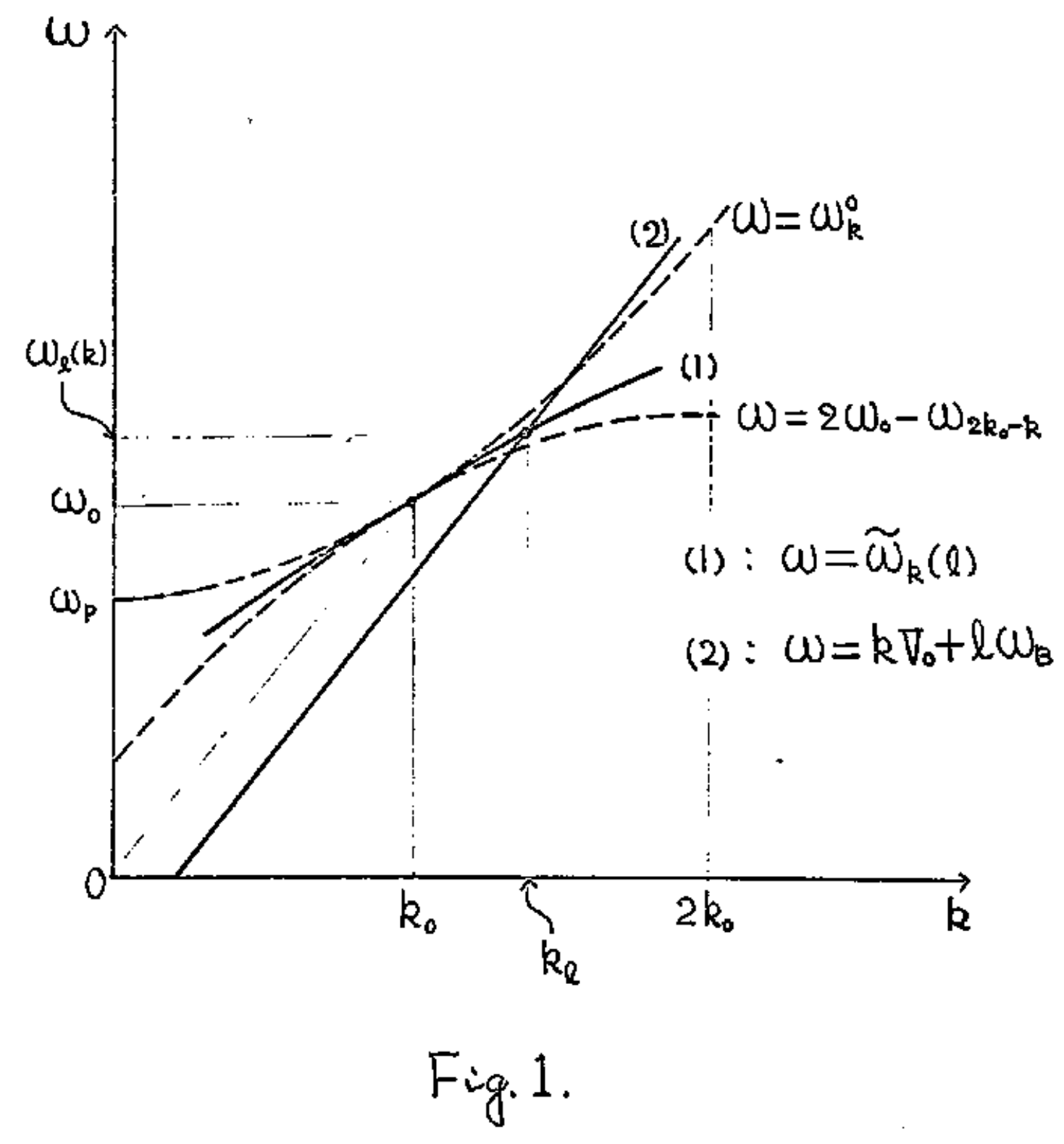

